\title{
Operating Procedures and Life Cycle Documentation for the Initial Graphics Exchange Specification
}

Greg F. Morea

IGES Project Manager

Ed Reid

Curtis Parks

Editors

Version 2.0

June 22, 1995
U.S. DEPARTMENT OF COMMERCE

Technology Administration

National Institute of Standards

and Technology

Electronics and Electrical Engineering Laboratory

Electricity Division

Gaithersburg, MD 20899

QC 



\section{Operating Procedures and Life Cycle \\ Documentation for the \\ Initial Graphics \\ Exchange Specification}

Greg F. Morea

IGES Project Manager

Ed Reid

Curtis Parks

Editors

Version 2.0

June 22, 1995
U.S. DEPARTMENT OF COMMERCE

Technology Administration

National Institute of Standards

and Technology

Electronics and Electrical Engineering Laboratory

Electricity Division

Gaithersburg, MD 20899

June 1995

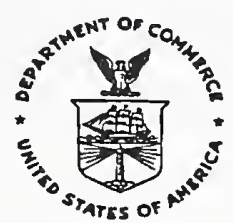

U.S. DEPARTMENT OF COMMERCE Ronald H. Brown, Secretary

TECHNOLOGY ADMINISTRATION

Mary L. Good, Under Secretary for Technology

NATIONAL INSTITUTE OF STANDARDS

AND TECHNOLOGY

Arati Prabhakar, Director 



\section{PREFACE}

The methods detailed in this document have provided the Initial Graphics Exchange Specification (IGES) community with advanced visibility of the changes approved by the voluntary organization responsible for the IGES Standard. Through this process, each technical change reaches consensus separately, and is held for incorporation into the subsequent version of IGES. These methods were adapted from the aerospace "engineering change proposal and change order" process. The process is still being improved through the use of hypertext on the Internet, thereby providing the organization with a timely response to proposals for extensions and repairs needed by implementors and users of IGES.

The implementation details of the process have evolved over the years, and now embody a complete life cycle system of electronic procedures. These procedures involve the $\operatorname{IAT}_{\mathrm{E}} \mathrm{X}$ publishing language, Internet submission/notification, and hypertext on-line information database $^{1}$ that is availability during IGES Organization meetings. The database includes an index of all changes formally approved, from the initial use of the process in 1981 to the present. All the database files may be searched to identify prior changes as well as the present status of each proposed change. The process additionally provides an audit trail for the consensus and quality checks, in both electronic and paper form, for each version and for each proposed technical change.

The process for integrating the approved changes electronically into the files constituting the "next version of IGES" was developed by Dr. Philip Kennicott of Sandia National Laboratories. The integration process enabled the use of the Internet for dealing with changes that were inherently in electronic form. This integration provided the means for placing approved changes directly into the Standard without re-entry (and possible errors) during production of an IGES version.

Examples of the use of the Internet include an automated distribution of Request for Change (RFC) documents, and World Wide Web access to RFC documents in-work by committee (http://www.eeel.nist.gov/iges) and the Edit Change Orders that constitute the next version of IGES (http://www.eeel.nist.gov/iges/ecoList.html). Each are updated following each IGES Project meeting.

Lastly, the authors developed the process for recording the committee actions and project consensus in the hypertext-files environment. Taken together and detailed in this document, the process documents the complete life cycle in an open environment.

1. The examples of the change process forms which appeared in appendices of the previous edition of this document (dated March 1, 1993) have been published in C. Parks, "Applying Hypertext to Managing Versions of a Standard," September 1993, NISTIR 5245; available from the IGES/PDES Office, NIST, Bldg 220, Rm. A-127, Gaithersburg, MD 20899. 


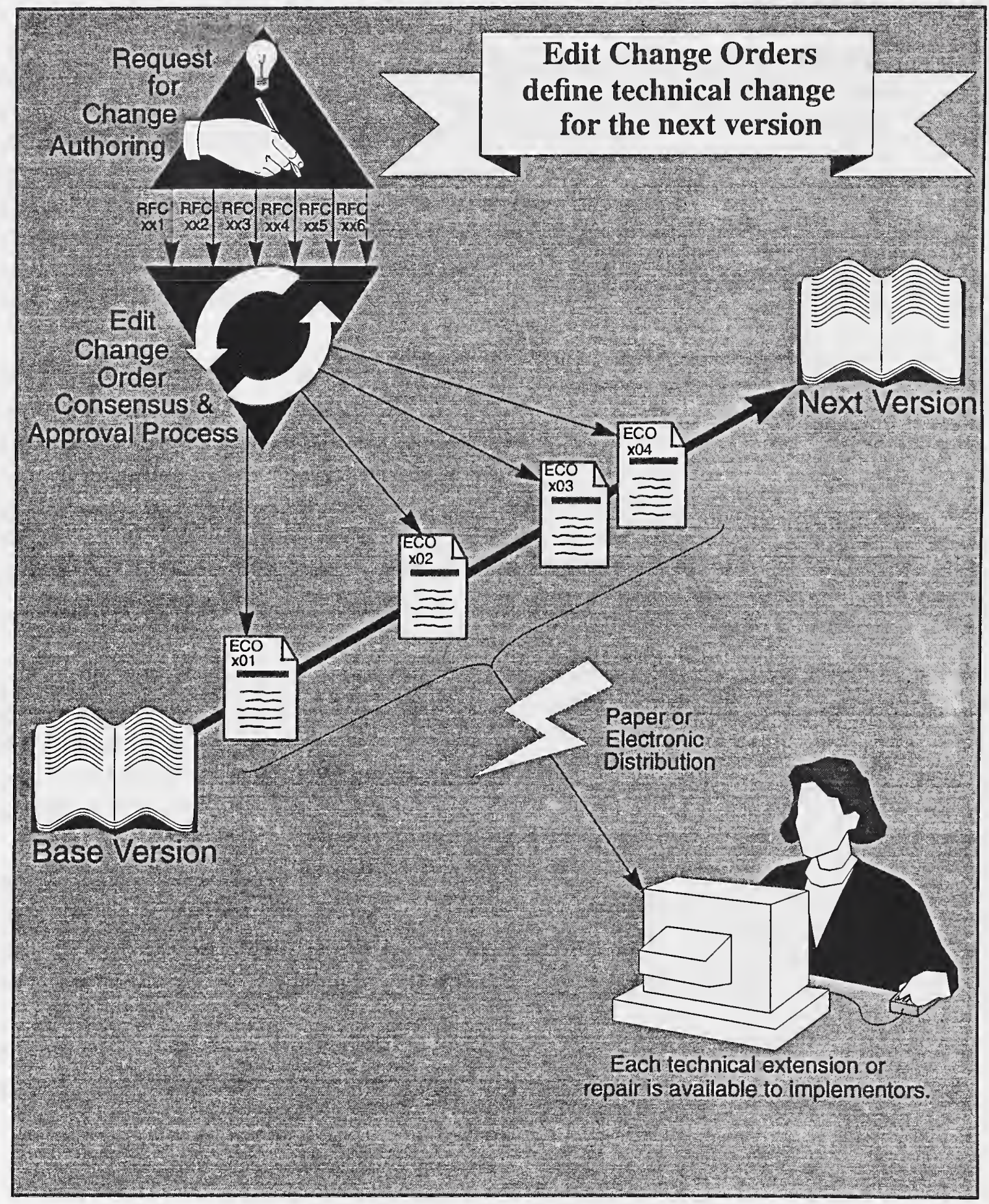

\section{Preface Illustration.}

The process for changes to IGES adopts the change management followed by industry. Industry configuration management process provides continuous product quality improvement over production runs. The result is timely response to technical change requests needed by the IGES community. The adopted change management practice is augmented by the publishing business practice of proofing prior to printing each version of the Standard. 


\section{Contents}

1.0 Introduction 1

1.1 Due Process and Consensus $I$

1.2 General Operating Policies for IGES Committees 2

2.0 The Initial Graphics Exchange Specification 3

2.1 The Role of the Specification 3

2.2 Changes to the Specification 4

2.3 Constraints for Changes To the Specification 5

3.0 The Processes for Approving Changes to the Specification 6

3.1 The Parts of a Request for Change (RFC) 6

3.2 The Technical Coordination Process 7

3.2.1 Origination of A New RFC 7

3.2.2 IGES Project Committee Coordination 9

3.2.3 Custodian Committee Coordination 10

3.2.4 The RFC Form - Guidelines and Electronic Aspects 15

3.3 The RFC Ballot Process 18

3.3.1 Oversight Review for Ballot Approval 18

3.3.2 The RFC Ballot 19

3.3.3 Ballot Summary and Ballot Evaluation 20

3.3.4 Ballot Follow-On Work in Custodian Committees 21 
3.4 The Gray Page Testing Process 24

3.4.1 The Gray Pages 25

3.4.2 The Gray Page Committee 26

3.4.3 Oversight Review for Gray Page Testing Approval 27

3.4.4 Existing Gray Page Material Recommendations 27

3.5 The Edit Change Order (ECO) Process 27

3.5.1 The Makeup of an ECO 28

3.5.2 Oversight Review for ECO Approval 28

4.0 The Process for Approving Major Changes to the Specification 30

4.1 New Versions of the Specification $\mathbf{3 0}$

4.2 Application Protocols 31

5.0 The Appeals Process 32

5.1 Definition of Appellants 32

5.2 Appeals procedure 32

6.0 IGES Change Tracking 33

7.0 Informal Forums for IGES Issues, Analyses, etc. 33

8.0 Step-by-Step User Guide to the RFC Process 34

8.1 Normal processing 36

8.2 Expedited processing 39

9.0 Editing, Managing, and Publishing the Specification 40

9.1 Editorial Review 40

9.2 Managing the document 40

9.3 Publishing the document 41

Appendix A, Activity Model: Changing IGES A 1

Appendix B, Example Cover Sheet Forms B 1 


\subsection{Introduction}

The purpose of this document is to provide a record of the underlying rationale and the detailed procedures by which the initial Graphics Exchange Specification (IGES) is changed, new versions are approved, and the document itself is edited, maintained, and published by the IGES Project of the IGES/PDES Organization (IPO).

It is intended that the Initial Graphics Exchange Specification continue to be submitted to and approved by the American National Standards Institute (ANSI) as a voluntary American National Standard.

This document also defines the life-cycle documentation supporting these activities and provides guidance for its use, including record keeping requirements, to provide evidence of compliance with these procedures. These records include Request for Change (RFC), Edit Change Order (ECO), and ballot results and comments.

Procedures given in this document are intended to be supplementary to and consistent with the documented procedures of the General Assembly of the IPO for the IGES Project. In general, procedures in this document are at a more detailed, working level. A function model is provided in Appendix A.

Several list formats are used in this document. Numbered lists indicate ordered items, bulleted lists indicate unordered items, cate required action items.

\subsection{Due Process and Consensus}

The primary conditions to be satisfied in changing and approving new versions of the Specification concern due process and consensus. Adherence to these procedures will result in the conditions for due process and consensus being met as new versions of IGES are developed and approved within the IGES Project of the IPO.

Due process means that any person (organization, company, government agency, individual, etc.) with a direct and material interest in the Specification has a right to participate once membership requirements are met by expressing a position and its basis, having that position considered, and appealing if adversely affected. Due process results in requirements that must be satisfied in developing consensus such as openness (participation open to all persons directly and materially affected), balance (no domination by any single interest category), and prompt, thorough consideration of written views and objections, with a concerted effort being made toward their resolution.

Consensus means that "substantial agreement" is achieved among those persons who are:

- $\quad$ affected by the matter at hand,

- $\quad$ entitled to determine its outcome, and

- participants in the decision process.

In this procedure, substantial agreement resulting in consensus can be obtained only after credible minority positions have been reconciled with the majority. (Section 5.0 outlines appeal procedures covering situations in which a minority position is unable to be reconciled.) 


\subsection{General Operating Policies for IGES Committees}

For the purposes of this document, IGES committees include the IGES Project Committee, IPO Technical Committees involved in work to change and approve new versions of the Specification, and committees established within the IGES Project of the IPO at the discretion of the Project Manager (such as the Gray Page Committee).

IGES Committees typically meet at IPO meetings. Specifics of upcoming meetings ${ }^{1}$, including preliminary agenda, are contained in the Meeting Announcement mailed to IPO members prior to each meeting. Interim meetings of the IGES Project and of IGES Technical Committees may be convened as necessary. Interim meeting announcements shall also include an agenda. Decisions about agenda items that are made at interim meetings shall have the same authority as decisions made at IPO meetings; decisions concerning "non-agenda" items of a non-trivial nature shall be deferred to the next meeting unless

- the item is urgent, and

- the number of attendees voting for approval is equal to or greater than a majority of the total committee membership of record.

Committee members who cannot attend any announced meeting may vote or comment on an agenda item by writing to the committee chairperson.

Minutes will be recorded for each official meeting of an IGES committee and will be made available to any interested person. At an official meeting, minutes of the previous meeting will be approved by vote. Minutes shall include sufficient detail to enable those familiar with the work of the committee to understand the business conducted.

Minimum requirements shall include:

a list of attendees

$\ll$ text of motions and names of all members making and seconding them

results of all votes taken

$\gtrless_{0}$ identification of all actions taken by acclamation.

In addition, for each RFC discussed, the RFC number and a summary of key discussion points, including names of participating members, should be included. Refer to Guidelines For Preparing Meeting Summaries in The Handbook For Chairs of IPO Technical Committees And Interest Groups.

At any duly called meeting of an IGES Committee, the agenda is left to the discretion of the Chairperson, since the topics to be discussed may depend on qualified persons being present. A vote always shall be taken on technical matters, and consensus is required. Either a vote or acclamation may be used for administrative matters, and consensus is not required. All actions shall be recorded in the minutes, including names of persons making and seconding motions when used, as well as key discussion points to document the reasons for the action taken.

Anyone with voting membership status in the General Assembly of the IPO may vote in any Technical Committee on any IGES matter. Membership in the IGES Project Committee is com-

1. Meeting announcements and registration information are mailed prior to each meeting; contact USPro, (703) 698-9606 or see World Wide Web page at http://elib.cme.nist.gov/nipde/. 
prised of the IGES Project Manager, the Deputy Project Manager, the Chairperson of every IPO Technical Committee involved in the project, the IGES Editor, the IGES Ballot Coordinator, the Change Control Secretary, the heads of IGES Project Subcommittees, and others at the discretion of the IGES Project Manager. A Technical Committee is "involved" if its scope of work as described in the Handbook (mentioned above) explicitly mentions IGES-related work. [Refer to IGES/PDES Organization Reference Manual (available from the IPO Office or at IPO meetings), for more information.]

In many instances in this document, the reference is made to Chairperson of the custodian committee, meaning the Chairperson of the relevant Technical Committee of the IPO General Assembly. In these instances, "Chairperson" can be interpreted to mean "Chairperson or appropriate designee." For several Technical Committees, the Deputy Chairperson has been given responsibility for coordination with the IGES Project Committee.

\subsection{The Initial Graphics Exchange Specification}

The Initial Graphics Exchange Specification defines a non-proprietary format for CAD system data. The format is used primarily for the exchange of data between different CAD systems. The fundamental unit of IGES data is an IGES entity type instance. The fundamental unit of communication of IGES data is an IGES file. The Specification therefore consists primarily of a collection of entity type definitions together with instructions for assembling a valid IGES file. "The Specification" refers to a document including several appendices. The main body of the document contains the official portion. The appendices are normally not part of the official portion.

Software units generally termed "processors" are used to read and write IGES data. "Pre-processors" write IGES data, and "post-processors" read IGES data. The term "translator" also is used when the conversion operation between the IGES data and CAD system data is to be emphasized.

Changes to the Specification may vary from a simple language clarification to a fundamental extension such as the addition of a new entity type. Extensions to the Specification occur primarily in response to new requirements identified by its users. Changes and extensions are subject to an IGES Project policy termed "Gray Pages." Changes and extensions subject to this policy are released from the Gray Page designation when tested and found suitable for general use.

\subsection{The Role of the Specification}

The Specification should be able to codify CAD system data accurately and completely for the purposes of successful exchange with other CAD systems, archiving, and sharing with other applications. Due to the diversity of the systems involved, perfect results under all circumstances are unlikely; however, it is intended that coverage of the CAD system data should be as complete as is practical.

Many types of entities are defined in the Specification, and may be used individually or collectively in order to accept the CAD data. There is a great amount of flexibility built into the Specification for accomplishing this. For example, the Associativity entity type provides flexibility for collective use of entities, and the Property entity type provides flexibility for modifying or providing additional information concerning another entity. The meaning of certain forms of these entity types is defined in the Specification, while for other forms, the meaning can be tailored to the individual situation and specified in the file itself. 
Careful choices must be made to ensure that the IGES entities used faithfully represent the important characteristics of the CAD system data (e.g., the visual, geometric, functional, and associative characteristics). Full usage of the flexibility of the Specification in representing data can maximize the potential for complete exchange results, but it must be emphasized that more complex entities should not be used when simpler entities are sufficient.

\subsection{Changes to the Specification}

Technical changes to the Specification are approved via a ballot process. The Request For Change (RFC) is the formal mechanism by which changes are proposed and balloted. Editorial changes to the Specification may be initiated by the IGES Project Committee without an RFC. In either case, the Edit Change Order (ECO) is the formal mechanism by which the IGES Editor is

- informed of the content of a change to the Specification (either main body or appendices), and

- authorized to make the change to the master copy of the Specification.

Figure 1 illustrates the major processes for changing and maintaining the Specification.

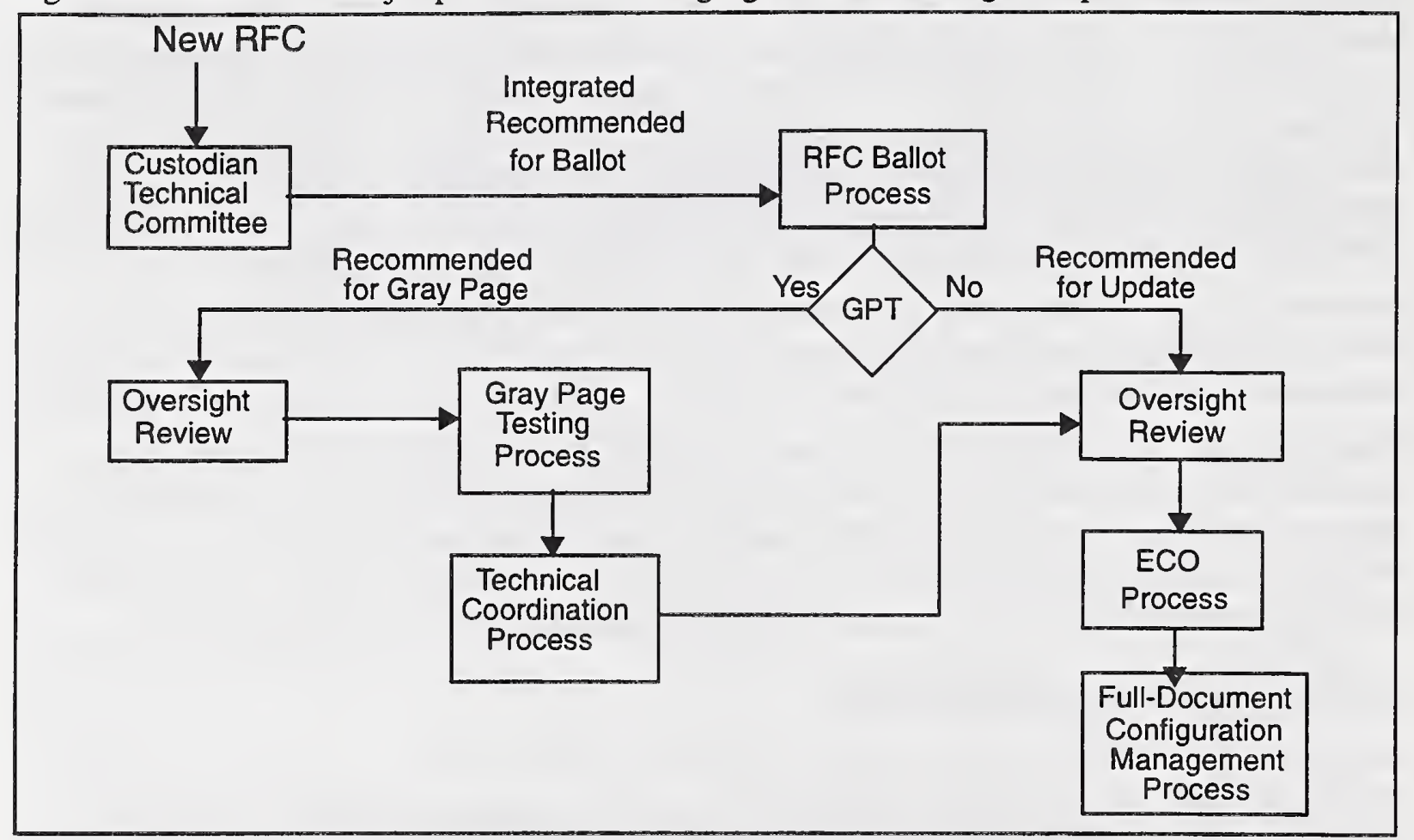

Figure 1. The Major Processes for Changing and Maintaining the Specification

Changes are divided into three types:

- Editorial: approval of the proposed change by the IGES Project Committee results in an ECO to direct the IGES Editor to change the Specification

- Clarifications or fixes: approval of the RFC from the ballot process means that the RFC content will become part of the main body of the Specification (subject to editorial harmonization)

- Extensions: approval of the RFC from the ballot process signifies approval of the concept involved, but the RFC content will become part of the Untested Entities Appendix ("Gray 
Pages”) until satisfactorily tested.

Editorial changes proposed are intended to correct misspellings, grammar problems, inconsistent style, etc., to repair errors in updating the Specification, and to improve utility of the document. Editorial changes do not require an RFC nor a mail ballot because they do not alter the intent of the portion of the Specification they affect.

Clarifications or fixes to "refine" the Specification are encouraged, because one goal is that the Specification be written clearly and succinctly to ensure consistent interpretation. Consistent interpretability should not depend upon the reader's attendance at IPO meetings. This category of change is distinguished from the "Editorial" category by its alteration of the intent of the portion of the Specification affected (e.g., adding a sentence to cross-reference related information is editorial-RFC not required; adding a sentence to specify entity usage is clarification-RFC required). This category also includes changing the Specification to "deprecate" an entity by moving it from the main body of the Specification to the Obsolete Entities Appendix; although such entities remain valid, new uses of them are not recommended.

Extensions generally involve adding new capabilities or making fundamental changes to the Specification (e.g., the addition of a new entity type or the addition of a new form of an existing entity). Most extensions must be tested to ensure they can be implemented correctly before they can become part of the main body of the Specification; this is called "Gray Page" testing and must include successful exchanges involving at least three processor implementations. See Section 3.4 for more information concerning the Gray Page process. At the IGES Project Committee's discretion, Gray Page testing may be omitted for "functional" extensions of mechanisms already proven in commercially available IGES processors (e.g., adding a new attribute to an existing attribute table entity, adding new text or line fonts) because the only result would be to identify that implementation actually occurred. Given the large number of existing untested entities to be tested, such a diversion of limited testing resources cannot be justified for so little value. Furthermore, since the decision to test also is balloted, anyone objecting to omission of testing may cast a disapproval vote.

\subsection{Constraints for Changes to the Specification}

A principal constraint regarding changes to the Specification has to do with upward compatibility. Upward compatibility is interpreted to mean that "old" files, i.e., files valid with respect to earlier versions of the Specification, should remain valid with respect to later versions of the Specification. The benefits of upward compatibility are that processors based on later versions of the Specification are able to accept files produced by processors based on an earlier version and that archived files will not become obsolete.

Upward-incompatible changes generally will not be allowed unless there is a clear case where the best interests of users of the Specification are served by approving them (e.g., the Specification may need to be tightened technically because what is allowed currently is counterproductivethis happened with the Conic Arc entity). A factor in considering upward-incompatible changes is the state of processor implementations as best they can be determined. In theory, adverse effects from upward-incompatible changes would be minimized if processors used the Global Section version number properly because it identifies if a file was created before or after the upwardincompatible change. In practice, this technique can fail because pre-processors may specify a 
later version number for a reason other than making the upward-incompatible change(s) required for that version.

Downward compatibility is interpreted to mean that processors based on earlier versions of the Specification are able to accept files produced by processors based on a later version because their robust design deals "gracefully" with extra data they do not understand. However, they cannot reasonably be expected to accept correctly files containing upward-incompatible changes because they will not be aware of them. Downward-incompatible changes generally are allowed; often they are unavoidable. However, to minimize costly interoperability problems, all changes to the Specification should be designed for as much version compatibility as is practical.

\subsection{The Processes for Approving Changes to the Specification}

Technical changes to the Specification are formally proposed, considered, and balloted by means of the Request for Change (RFC) process. Editorial corrections (e.g., typographical errors) may be handled by notifying the IGES Editorial Committee without using the formal RFC process.

An RFC which ultimately results in a technical change to the Specification may involve the generation and retention of a considerable amount of information over a period of time; however, the initial stages of originating and proposing an RFC purposely are kept simple to encourage potential RFC authors.

RFCs are administered by the IGES Project Committee. The work to take a potential RFC from concept to completion is coordinated by a technical committee (e.g., Drafting) designated as custodian.

RFCs are balloted within the IPO General Assembly after approval by custodian committee(s) and the IGES Project Committee. An RFC's problem statement and proposed solution should demonstrate the author has 1) identified all implications of the proposed change to the Specification, and 2) prepared a clear, succinctly written proposal. Past experience reinforces the need to do a complete, high-quality job initially; otherwise, considerable time and effort will be expended during revisions that result from the balloting process.

A later section explains more informal ways of presenting IGES-related ideas, issues, questions, etc., to the IPO General Assembly membership for their written comments.

\subsection{The Parts of a Request for Change (RFC)}

A complete RFC consists of three parts: an RFC Cover Sheet, an RFC Form, and an RFC Folder. Each of these parts evolves as the RFC traverses the various coordination processes.

The RFC Form is the heart of the complete RFC. It originates with the RFC author and encompasses the technical details of the proposed change. The RFC Form is circulated as necessary. All technical deliberations are made on the basis of the content of the RFC Form. RFC ballots are cast against the content of the RFC Form. The RFC Form includes limited administrative information which is either required for managing associated processes or considered to be relevant general information.

The RFC Cover Sheet is an administrative tool originating with the IGES Change Control Secretary. It is used to record all official business transactions that unfold concerning a particular proposed change (e.g., Form log-in date, Technical Committee decisions, IGES Project Committee 
approvals). The Cover Sheet is tied to the RFC Form but is not as widely circulated as the Form. Responsibility for the Cover Sheet begins with the Change Control Secretary, then responsibility changes for various entries through the RFC process.

The RFC Folder originates with the custodian committee. It is an open-ended storage device for technical records pertaining to the RFC, such as ballot comments made on the RFC and associated records for custodian committee resolution of these comments. The RFC Folder remins with the custodian committee.

See Appendix B, Example of Cover Sheet Forms. The blank RFC Cover Sheet may usec, out is not initially required; the RFC may initially be in any legible format. The author is encouraged to create the electronic form of the RFC as soon in the process as possible, and prior to assigning a tracking number. The "skeleton file" for use ${ }^{2}$ with any text editor together with its README file may be obtained from IGES Project Officers or through a link displayed on a World Wide Web page (http://www.eeel.nist.gov/iges/igesTools.html). Section 3.2 documents the meaning and collection of the data.

RFCs accepted into the coordination process are numbered sequentially. Sequential "issue" versions ("A," "B," "C," etc.; where " $\mathrm{A}$ " is the first revision) of each RFC may exist to track changes introduced during the coordination process.

All RFCs will exist officially as paper documents. RFC Cover Sheets and RFC Forms will be archived by the Change Control Secretary as paper documents. All issue versions will be archived as separate documents.

Ultimately, the master copy of the RFC Form will exist and be maintained in electronic form (currently the $\mathrm{LAT}_{\mathrm{E}} \mathrm{X}$ format, which is basically ASCII text). Further details appear later in this section.

\subsection{The Technical Coordination Process}

This section defines the process by which new RFCs are received, logged, assigned to a custodian committee for technical coordination and approval, and prepared for ballot.

\subsubsection{Origination of a New RFC}

Anyone may propose a change to the Specification, but it should be kept in mind that an RFC is intended to document both the need to make a change, and a carefully prepared proposal to accomplish that change. Problems without apparent solutions or with incomplete solutions should be addressed initially by one of the other methods. Possible methods are suggested in Section 7 identifying another way to deal with the problem, or confirming the need to create an RFC using suggestions of others to formulate the proposed solution.

Consideration of a proposed change is initiated by furnishing the IGES Change Control Secretary with a technical description of the proposed change in the form of a new RFC. A new RFC must contain the minimum information and structure listed below before the secretary will assign a number; however, most authors supply a "first draft" of the complete RFC. New RFCs supplied to the Change Control Secretary must be in paper form; preparing the electronic form at this stage is

2. The details of editing in $\mathrm{LAT}_{\mathrm{E}} \mathrm{X}$ are beyond the scope of this document; the RFC author is encouraged to seek documents on the subject, such as L. Lamport, $I A T_{E} X$ A Document Preparation System, 1986, Addison-Wesley Publishing Company. 
optional. If attempting to expedite the RFC, it is permissible to take the RFC to the point of "Recommended for Ballot" before getting the number assigned (see Section 8.2).

To prepare a new RFC, choose one of these methods:

- Manual form production: Photocopy the RFC form in Appendix B. Type, or hand print clearly - using only black or dark blue ink, or prepare text with a word processor-information defined below and in 3.2.4. Alternatively, use any word processor or text editor to simulate the appearance of the RFC form; however, this will cause rework later since both the RFC form and the portion of the Specification to be changed must be prepared in "integrated electronic form" using the Specification document processor before the RFC will be included in any mail ballot.

- Integrated Electronic form production: Refer to section 3.2.4 for requirements of electronic form RFCs. Prepare RFC in conformance with all requirements; requests for exceptions to the requirements routinely are disallowed. Save to floppy disk, print a paper copy, and forward both to the IGES Change Control Secretary. CAUTION: the secretary will assign an RFC number based on the paper copy without checking the electronic form for conformance with section 3.2.4 requirements. However, non-conforming RFCs will not be included in any mail ballot. The RFC author and the custodian committee jointly are responsible to ensure complete conformance. The IGES Editorial Committee may be consulted for assistance, but is not responsible for doing this work.

\section{Minimum information requirements for a New RFC are:}

1. Name, mailing address, and telephone number of the author of (or contact for) the new RFC.

2. Optionally, for RFCs extending the Specification, identification of two or more systems having a similar capability.

\section{Minimum structure requirements for a New RFC are:}

1. RFC Title - Short but descriptive.

2. Problem and impact statement - A statement expressing the shortcoming or error in the current Specification, along with an indication of the impact of this problem.

3. Proposed solution - A technical description of the change to the Specification that is being proposed, along with an explanation of how this is a solution to the problem described.

\section{General Guidelines For Originating RFCs are:}

1. Two or more entities that are related to each other must be included in a single RFC. When a new entity must point to another new entity from its Directory Entry, Parameter Data, or Additional Pointers lists, it is counterproductive to separate them because confusion will result if one RFC is approved and the other is not.

2. The problem statement and proposed solution should clearly identify affected passages by (a) IGES version number, (b) section number, and (c) page number. For example, use a phrase such as "IGES Version 5.0, Section 4.59, third paragraph on page 226." All RFCs must refer to the latest available version of the Specification when they are initiated.

3. Entities should be referenced by both type and form numbers. There must be a distinction between the instance of an entity and its name; names are proper nouns and are capitalized 
(except for the word "entity"). For example, "... the Witness Line entity (Type 106, Form 40)..." is a reference to the name, while the phrase "...the visible segments of a witness line..." is a reference to an instance of the entity. When adding one new type, the entity type should be specified as the RFC number (without suffix) assigned by the Change Control Secretary, and the numbering of form $(s)$ should be ascending starting at 1 . (The permanent type number will be assigned by the IGES E tor when the change is incorporated into the Specification.) The RFC Form's proposed solution should indicate the numeric range (e.g., RFC 999D might use "Add the Essential entity (Type 999, Forms 1-20) in the 300-series of entities"). If one form is being added to an existing entity type, the entity form should be specified as the RFC number (without suffix). When adding multiple new types or multiple new forms to existing types, use a unique alphabetic prefix to the RFC number (e.g., RFC 987B might use "Add the Magnificent Definition entity (Type A987, Forms 1-5) in the 300-series and the Magnificent Instance Entity (Type B987, Forms 1-5) in the 400-series, and add forms C987-E987 to the Point Entity (Type 116)"). If changing the Global section, use a phrase such as "next available 3 parameters." If the RFC is so complicated that the above guidelines might cause more confusion than clarification, consult the IGES Editor, Change Control Secretary, or the IGES Project Committee for guidance.

\section{$\underline{\text { RFC Cover Sheet Information Recorded During This Stage: }}$}

Date the new RFC was received.

RFC Number assigned.

RFC Title.

Name, mailing address, and telephone number of the author of the new RFC.

When supplied, identification and attachment of the electronic form of the new RFC.

\section{RFC Actions Taken During This Stage:}

1. The Change Control Secretary examines new RFCs received for adherence to the minimum requirements. Return contact is made with authors of new RFCs that do not meet the minimum requirements so they may be resubmitted.

2. The Change Control Secretary originates an RFC Cover Sheet for each new RFC meeting the minimum requirements.

3. The Change Control Secretary archives each RFC Cover Sheet and the associated documentation making up the original RFC.

\subsubsection{IGES Project Committee Coordination}

The Change Control Secretary delivers all new RFCs to the IGES Project Committee in a timely manner. This means all new RFCs collected since the previous meeting of the IPO General Assembly are distributed beforehand to this committee in preparation for consideration at the following meeting.

At the IPO meeting, the IGES Project Committee examines each new RFC and decides whether or not Gray Page ${ }^{3}$ testing is required; these data appear on the integrated RFC form. The IGES Project Committee designates a custodian committee to be responsible for the RFC. Technical 
Committees sharing an interest in the RFC are termed "joint-interest committees" and also are identified to the extent possible at this time.

These actions are considered to be official business by the IGES Project Committee.

Typically, an RFC addressing a specific technical issue is assigned to an IPO Technical Committee on the basis of subject matter, while an RFC addressing a broad issue or having a broad scope relative to the Specification will be assigned to the Implementors' Committee or to the IGES Project Committee itself.

\section{RFC Cover Sheet Information Recorded During This Stage:}

Date of the IGES Project Committee meeting at which new RFC was first considered.

Indication of whether or not Gray Page testing is required, verify the same indication on the RFC Form.

Custodian committee assigned. Joint-interest committees assigned (if any).

Optionally, comments or recommendations to the custodian committee.

Optionally, for RFCs extending the Specification, identification of two or more systems having a similar capability.

\section{RFC Actions Taken During This Stage:}

1. Approximately one month before an upcoming meeting, the Change Control Secretary distributes a copy of each new RFC received to members of the IGES Project Committee for their review.

2. For each new RFC, the originally archived Cover Sheet is updated by the Change Control Secretary to reflect the determinations made during the IGES Project Committee coordination.

3. For each new RFC, the Change Control Secretary supplies the Chairperson of the custodian committee with the updated Cover Sheet, the associated documentation, and the electronic form if available.

4. For each new RFC, the custodian committee chairperson supplies the RFC author with a copy of the Cover Sheet received from the Change Control Secretary.

5. If Gray Page testing is required for the RFC, the Change Control Secretary supplies the head of the Gray Page Committee with a copy of the updated Cover Sheet and any associated documentation.

\subsubsection{Custodian Committee Coordination}

The custodian committee for an RFC is responsible for the technical coordination of that RFC. The outcome of the coordination activity, shown in Figure 2, is reported back to the IGES Project Committee for oversight review and final approval. Within custodian committees, technical coordination of new RFCs will be scheduled in as timely a manner as possible.

3. Beginning with IGES version 5.3, entries designated Gray Pages will not be in a separate section and will be flagged as untested. 


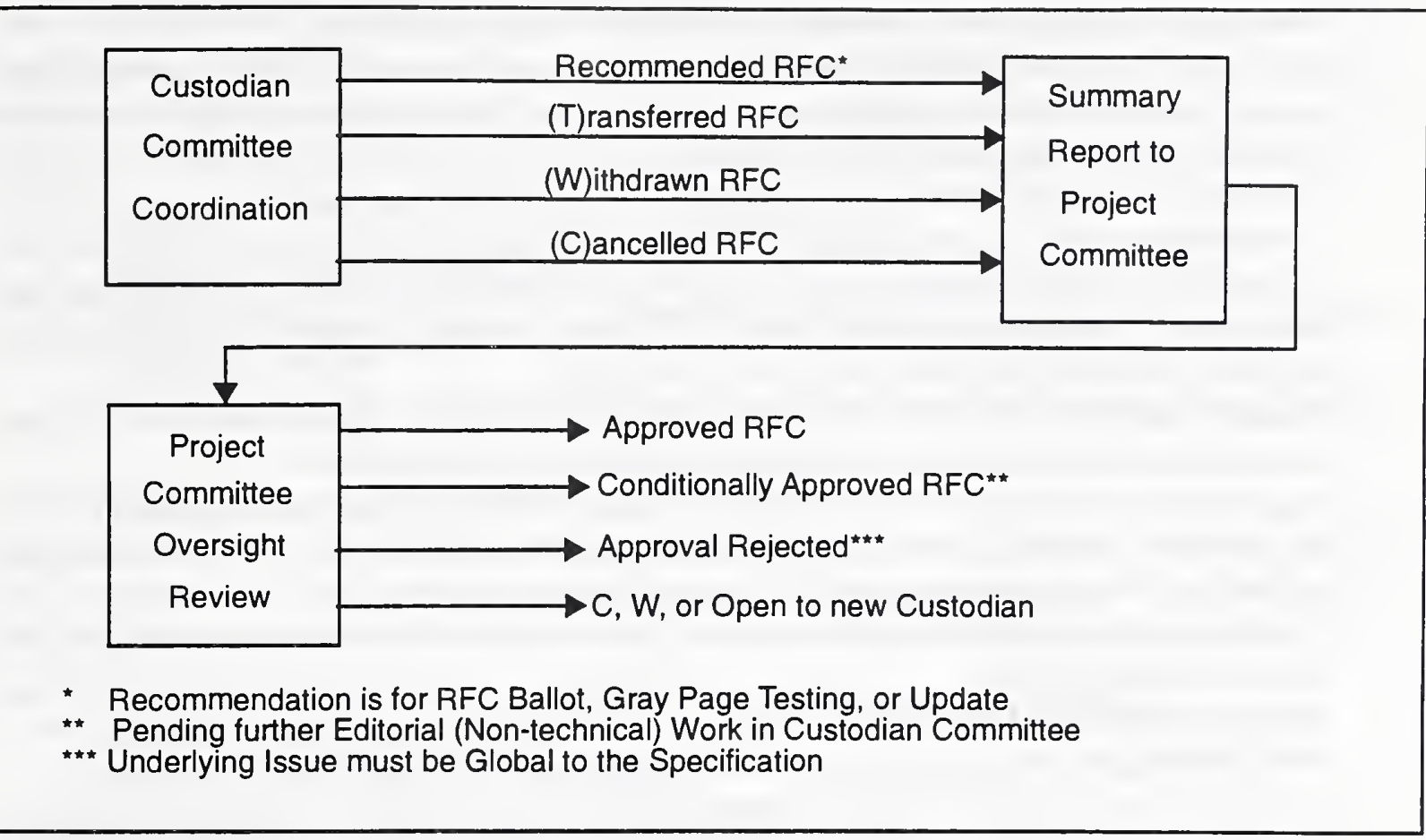

Figure 2. The Custodian Committee Coordination and Project Committee Oversight Review Process

The technical deliberations of the custodian committee may result in issuing a revision of the RFC. The custodian committee chairperson shall coordinate RFC revision process. He shall ensure that the reason for revision is entered on the cover sheet and that revisions are adequately distributed.

The custodian committee is responsible for managing the participation of joint-interest committees in the technical coordination of the RFC.

All balloted RFCs appear in their "integrated form," i.e., they accurately illustrate how the Specification would look should the proposed change actually be made (except that the type and form numbers may be temporary values based on the RFC number). Logically complete porticis of the Specification will be depicted. In general, this means full pages upon which changes occur. If the first and last page of a 30-page entity description change, common sense suggests only those two pages must be depicted, but if 2 pages in a 4-page description change, all 4 pages should be depicted. The IGES Editor and IGES Project Committee shall jointly determine what constitutes a "logically complete" depiction. Producing the integrated form of an RFC requires both the electronic masters and access to the document processor used to prepare the Specification for publication.

It is assumed that RFCs will exist in the "integrated electronic" format prior to the editorial review cycles identified below. Although technical assistance for this will be available through the IGES Editor and the Editorial Committee, custodian committees have the responsibility to see that RFCs are generated and maintained in the electronic format. With respect to technical coordination, an RFC will always be designated to belong to one of the following states: 
- Unassigned: RFC has been accepted by the Change Control Secretary but has not been assigned to a custodian committee by the IGES Project Committee.

- Open: RFC has been assigned to a custodian committee by the IGES Project Committee as an "active" work item of the custodian committee. The custodian committee is responsible for coordinating the RFC's advancement through the approval process.

- Tabled: RFC is being held by the custodian committee for future action.

- Transferred: RFC has been returned from the custodian committee to the IGES Project Committee for reassignment with Open status to a different custodian committee because the current custodian committee feels the RFC is not within its area of expertise; the IGES Project Committee may reassign or return the RFC.

- Recommended for Ballot: RFC has been reviewed by the custodian committee both technically and editorially, and the committee consensus favors approval. Approved for Ballot: A "Recommended for Ballot" RFC has been reviewed and approved by the IGES Project Committee and will be included in the next mail ballot provided the "integrated electronic form" is complete and correct.

Balloted: RFC mail ballot results have been received and forwarded to the custodian committee for coordination.

Recommended for Gray Pages: RFC has been affirmed by the voting and ballot comment resolution process, and the custodian committee consensus is that the proposal should be labeled as UNTESTED Appendix of in the Specification until testing requirements are completed.

- $\quad$ Recommended for Update: RFC has been affirmed by the voting and ballot comment resolution process, and the custodian committee consensus is that the proposal should be incorporated into the main body of the Specification immediately because no testing is required or the required testing has been completed. This status may be assigned immediately after balloting or following future successful Gray Page testing. Edit Change Order (ECO): A "Recommended for Gray Pages" or "Recommended for Update" RFC has been approved by the IGES Project Committee and will be incorporated into the next version of the Specification.

- Reviewed: RFC has received "oversight" review by the IGES Project Committee following the assignment of one of the "recommended" states by the custodian technical committee, but the IGES Project Committee rejects the "recommended" status for specific reason(s). The RFC is returned to the custodian technical committee to resolve the prob$\operatorname{lem}(\mathrm{s})$.

- $\quad$ Cancelled: RFC has been removed from further consideration by its custodian committee; this may occur before or after balloting. This status may be appealed to the IGES Project Committee if the author disagrees with the custodian committee's decision.

- Withdrawn: RFC has been removed from further consideration by its author; this may occur at any time subject to acceptance by the custodian committee (if withdrawal is to be rejected by the custodian committee, it must first identify an alternate author to assume responsibility for the $\mathrm{RFC}$ ).

The designation of these states (except Open, Approved for Ballot and ECO) is considered official business of the custodian committee. The designation of Open, Approved for Ballot and ECO states is considered official business of the IGES Project Committee. All state assignments must be promptly communicated to the IGES Change Control Secretary by the appropriate committee chairman. 
The custodian committee chairperson reports to the IGES Project Committee whenever one of the states recommended is reached (any of the three), Transferred, Withdrawn, or Cancelled. The report summarizes the history and present situation for the RFC. The IGES Project Committee exercises oversight review to ensure policies and procedures have been followed properly and to determine if there are any reasons why the status of the RFC being presented is inappropriate.

For Recommended RFCs, the IGES Project Committee will:

- $\quad$ approve the proposed status, or

- approve the proposed status on the condition that specified additional work be done by the custodian committee, or

- $\quad$ refuse approval and return the RFC to the custodian committee with "Reviewed" status.

The IGES Projec: Committee may refuse approval only on the basis of an issue that is of a global nature for the Spectication. The issue cannot be of a specific technical nature for which the custodian committee or a joint-interest committee could claim expertise. Sections 3.3.1, 3.4.3, and 3.5.2 address oversight review in more detail for each of the Recommended states.

For Cancelled or Withdrawn RFCs, the IGES Project Committee will either:

- approve the proposed status, or

- decide on an alternative course of action.

For Transferred RFCs, the IGES Project Committee will either:

- $\quad$ approve the status and appoint a new custodian committee to receive the RFC with "Open" status, or

- disapprove the status and send the RFC back to the same custodian committee with "Open" status. It is assumed that "Transferred" status will be assigned at the beginning of RFC consideration rather than after much work (such as balloting) has been completed.

The above actions are considered official business on the part of the IGES Project Committee.

For recommended RFCs, Figure 3 illustrates the relation among the technical coordination process, the editorial review process, and the IGES Project Committee oversight review process. The "Recommended for Ballot" state for an RFC implies that the iterative process depicted in the figure between the custodian committee and the IGES Editor has been satisfactorily completed. It is possible that the editorial review cycle will result in editorial changes to the RFC and a new version will need to be produced and recommended. Editorial corrections after balloting are not intended to result in repeat balloting. (This is consistent with the fact that any editorial corrections may be made at any time without balloting with approval of the IGES Editor and the IGES Project Committee.) 


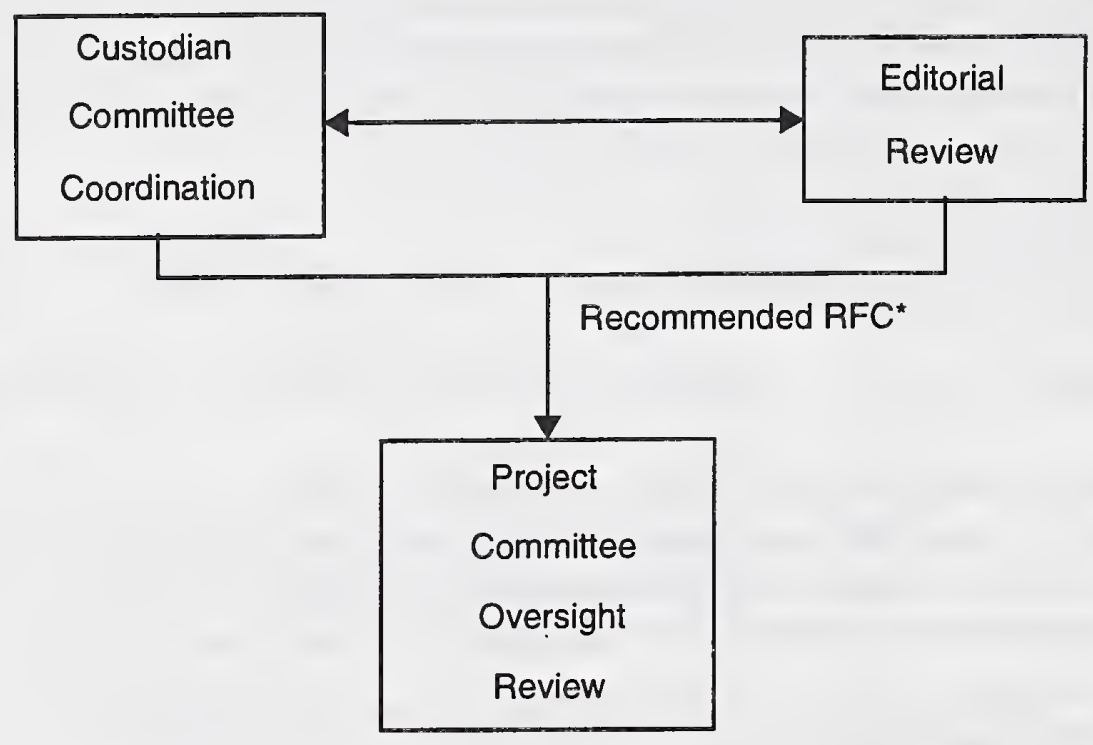

* Recommendation is for RFC Ballot, Gray Page Testing, or Update

Figure 3. Custodian Committee Technical Coordination and Editorial Review of RFCs

The objective of the editorial review cycle between the IGES Editor and the custodian committee is to come to a mutual agreement that the RFC is in the proper form, and that its statement is clear, succinct, and complete. This objective reflects the requirement that the proposed change to the Specification must not only improve the Specification, but must also be able to be easily and unambiguously evaluated at ballot time by the voting members of the IPO General Assembly.

It is the responsibility of the custodian committee to initiate and maintain momentum in the editorial review process.

Editorial Review Operating Principles:

1. Ensure that the General Guidelines For Originating RFCs, Section 3.2.1, have been followed.

2. Maintain consistency of style across RFCs.

\section{RFC Cover Sheet Information Recorded During This Stage:}

Identification (with associated dates) and brief description of the issue revisions of the RFC originating during the technical coordination process.

For RFCs Withdrawn, Transferred, Canceled, or Recommended:

Date action was taken

Issue revision for which action taken

Reason(s) for action (all except Approved)

Identification of accompanying electronic form (if Recommended for Ballot)

Identification (with associated dates) of joint-interest committee participation in the techni- 
cal coordination proces

Date the summary repo was made to the IGES Project Committee.

IGES Project Committee decision, along with reasons.

\section{RFC Actions Taken During This Stage:}

1. For each joint-interest committee named for an RFC, the custodian committee chairperson has the responsibility to supply a copy of the RFC to the chairperson of that committee and to manage the technical coordination of the RFC with that committee.

2. The custodian committee keeps the Cover Sheet up-to-date for each RFC for which it has custodianship.

3. The custodian committee chairperson supplies the Change Control Secretary with a copy of each issue revision of the RFC.

4. The custodian committee strives to write the RFC in accordance with the editorial review principles given above.

5. For Recommended RFCs, the custodian committee chairperson signs and dates the correct issue revision of the RFC Form.

6. The custodian committee chairperson reports to the IGES Project Committee whenever any one of the states Withdrawn, Transferred, Cancelled, or Recommended is reached and supplies the Change Control Secretary with an updated Cover Sheet and the latest issue revision of the RFC Form.

7. For each RFC reported, the Change Control Secretary accepts the latest issue revision of the RFC Form and the updated Cover Sheet from the custodian committee. The Secretary updates the Cover Sheet to reflect the IGES Project Committee decision and subsequently updates the archives.

\subsubsection{The RFC Form - Guidelines and Electronic Aspects}

This section examines the RFC Form, both as a paper document and as an electronic document.

\section{Guidelines for Completing the RFC Form:}

See Appendix B for a copy of a blank RFC Form. The contents of the RFC Form and their definitions are as follows.

Contents of the Cover Sheet are:

1. Date - Date the RFC was received by the Change Control Secretary

2. RFC Number - Sequential number assigned by the Change Control Secretary in the order RFCs are received

3. Title - Short, descriptive title for the RFC

4. Author - Name, mailing address, telephone number, and optionally FAX number and e-mail address of the person to be contacted about this RFC. If this person is not the author, also list the actual author's name.

5. Custodian TC - The IGES committee responsible for the RFC.

6. Gray Page Testing - Yes or No depending on whether or not this testing is required.

7. Issue Revision - A, B, C, etc. denoting the revision of this RFC.

8. Revision Date - The date work on this issue revision began. 
9. ECO Number - Signifies that "Recommended for Gray Pages" or "Update" status was upheld in oversight review by the IGES Project Committee.

10. Contents of succeeding pages (numbered $2-n$, and with the assigned RFC number in the header):

11. Problem Description - A statement expressing the shortcoming or error of the current Specification, along with an indication of the impact of this.

12. Proposed Solution - A technical description of the change to the Specification being proposed, along with an explanation of how this solves the problem described.

\section{Guidelines For The Electronic Form Of RFCs:}

Section 3.2.1 offers the option of submitting an RFC in electronic form ${ }^{4}$ (in addition to the required paper form) to obtain a number from the IGES Change Control Secretary. Although having the RFC in electronic form is optional for getting a new number, it is mandatory for getting the RFC to "Approved for Ballot" status; this policy has been adopted to reduce the problems caused in the past when the RFC wording was inconsistent with the Specification and the IGES Editor had to resolve the discrepancy.

The most important concept of the electronic form RFC is called "integration"; this means that the actual RFC text is inserted into a temporary copy of the Specification to which it applies. This portion can then be processed and printed to depict the actual appearance of the Specification as if the proposal were approved.

Integration offers significant advantages:

- $\quad$ consistency is assured because the actual text is changed;

- $\quad$ rework is avoided by making the change once;

- accuracy is improved by eliminating data reentry;

- comprehension is enhanced because voters see the proposal "in context" rather than as isolated editing instructions.

The disadvantages are:

- Work is shifted from the end of the RFC process to its beginning; therefore, this work is wasted if the RFC is not approved. (This may be an advantage since it encourages authors to research their change and identify support before going to the trouble.)

- $\quad$ Extra work may discourage authors from submitting useful RFCs

- Integration deemphasises deletions, so proposals that require only deletion may be less understandable than an editing directive such as "delete paragraph 1 on page 10."

- Preparing the "integrated" form requires access to the electronic master of the Specification as well as the document processing software

Version 5.1 of the Specification was prepared using $\operatorname{LAT}_{\mathrm{E}} \mathrm{X}$ (a public domain application) for the body text and IGESDRAW'M for IGES illustrations.

$\mathrm{IAT}_{\mathrm{E}} \mathrm{X}$ is called a text formatter because it uses markup symbols to specify what the document

4. The software products cited in this section are intended to indicate possible applications for use and are not necessarily endorsed by the authors or their employers. 
will look like when printed (e.g., IPAR marks the beginning of a paragraph and ISECTION indicates section title); this differs from word processors that typically display the page work area to look like its printed appearance.

IAT $_{\mathrm{E}} \mathrm{X}$ input is relatively easy to prepare, especially when limited changes are involved; even extensive new sections are manageable by starting with an existing section that is similar to the new information to be added. The IGES Editorial Committee will make portions of the Specification available upon request between meetings; electronic copies of the Specification also will be available on a personal computer at the IPO meetings. RFC authors without access to an appropriate computer between meetings should obtain assistance from the custodian committee's chairperson.

New RFC authors planning to prepare the electronic form should refer to existing RFCs. Examples may be obtained from the IGES Project to enable comparison of the visual appearance of an RFC Form to the IAT $_{\mathrm{E}} \mathrm{X}$ input that generated it. This will aid in understanding how to modify the LAT $_{\mathrm{E}} \mathrm{X}$ input to obtain desired results.

When prior arrangements have been made with the IGES Editor, the $\operatorname{LAT}_{\mathrm{E}} \mathrm{X}$ input text may be prepared in the native format of the originating word processor for the RFC (WordPerfect ${ }^{\mathrm{TM}}$, Microsoft Word ${ }^{\mathrm{TM}}$, etc.). In the absence of such an arrangement, the $\operatorname{IAT}_{\mathrm{E}} \mathrm{X}$ input should be ASCII text. When ASCII text is submitted, each line should be terminated by a "line feed" sequence $(<\mathrm{CR}><\mathrm{LF}>$ or $<\mathrm{NL}>)$. Lines should not exceed 80 characters. Paragraphs should be separated by a single blank line. In general, printing the text file on a monitor screen or a printer should yield results resembling how the final version should appear (with allowances for the text formatting markup symbols that are present).

Submission of new RFC material in electronic form to the Change Control secretary must use either e-mail or floppy disks. Internet submissions use an exploder (iges@eeel.nist.gov) to distribute and archive the submitted RFC. A floppy disk (any format or density) also is acceptable. Disks should be clearly labeled on their paper label with the author's name, the RFC number (if assigned), the operating system, and the density; otherwise, time will be wasted experimentally reading the disk to identify its format.

Material submitted in electronic form for an illustration within an RFC must be submitted as an IGES file unless the illustration cannot be depicted in IGES (e.g., a photograph); discuss use of any non-IGES artwork with the IGES Editor in advance of RFC preparation. Use one IGES file in ASCII (non-compressed) form for each illustration. Include only the illustration itself, without borders or captions. An illustration may be either full page [15x20 cm (6x8 inches) portrait] or half page [ $15 \times 10 \mathrm{~cm}(6 \times 4$ inches) landscape]. The file must be one-to-one scale. Text must be font 0 (standard block) and a minimum of $5 \mathrm{~mm}(0.2$ inch) in height (smaller text may not reproduce clearly). Text used for explanatory annotation should be $6 \mathrm{~mm}(0.25 \mathrm{inch})$. It is preferred, but not required, that all data be two-dimensional and defined in the XY plane, i.e., surface and solid entities should not be used. Dimension entities are acceptable. Any RFC author lacking access to a CAD system that can generate an acceptable IGES file should seek help from the custodian committee's chairperson or from the IGES Editorial Committee. 


\section{Ballot-Ready form:}

An RFC is in "integrated electronic form" (i.e., "ballot-ready") when:

$\bullet$

$\bullet$ editorial review of RFC content by the IGES Editorial Committee has been completed, both the RFC Form and proposed changes to the Specifications have been prepared for and processed correctly by the designated document processor for the Specification (currently $\operatorname{LAT}_{\mathrm{E}} \mathrm{X}$ ), the designated IGES post-processor for the Specification (currently IGESDraw ${ }^{\mathrm{TM}}$ ), printed output has been produced from the electronic input for delivery to the Change Control Secretary along with the e-mail or floppy disk(s) containing the electronic input.

\subsection{The RFC Ballot Process}

This section specifies the process by which RFCs are given final approval for ballot and balloted within the IPO General Assembly. This section also specifies how ballots are summarized and evaluated, and how individual ballot comments are handled.

\subsubsection{Oversight Review for Ballot Approval}

The IGES Project Committee performs oversight review for ballot approval on each RFC reaching "Recommended For Ballot" status upon request of the custodian committee's chairperson, who has verified the completion of these actions:

- $\quad$ RFC content has been approved by the IGES Editorial committee.

- Custodian committee has reached consensus on approval of the RFC for ballot.

The request for oversight review is a report containing at least the following:

1. A high-level (i.e., the "gross differences") comparison between the RFC originally submitted by the author and the RFC that has reached "Recommended For Ballot" status if significantly changed.

2. The role of joint-interest committees and the results of their participation.

3. A summary of how consensus was obtained.

4. The Gray Page Test Plan, when this testing is required. See Section 3.4.1.

\section{Ballot Oversight Review Operating Principles:}

1. An RFC shall be capable of being considered and balloted as an independent, self-contained item. It shall not depend upon other RFCs not yet in final ECO form for its validity, its correctness, or its completeness.

2. An RFC should not conflict with or duplicate another RFC in any stage of processing. (A context-searchable index of all RFCs is available at IPO meetings or by request to the Change Control Secretary.)

3. There should be technical consistency across RFCs.

Options for the IGES Project Committee following oversight review were given in Section 3.2.3. If all criteria are met, the RFC is assigned "Approved for Ballot" status by the IGES Project Com- 
mittee; this status may be made conditional on completion of procedural requirements such as minor editorial corrections.

If the oversight review results in an RFC approved or conditionally approved for ballot, a ballotready form of the RFC must be supplied to the Change Control Secretary. In many cases, because of the editorial review that has occurred, the RFC as presented for oversight review already will be ballot-ready. However, if this is not possible at the time of oversight review, or if the oversight review itself results in editorial changes, the custodian committee chairperson can make arrangements with the Change Control Secretary for supplying a ballot-ready form of the RFC at a later date. (The custodian committee chairperson decides whether to return the ballot-ready form to the committee for final review; the RFC need not return to the IGES Project Committee unless the custodian committee decides to change it again.) Arrangements made with the Change Control Secretary must take into account the Secretary's responsibility to supply the Ballot Coordinator with all ballot-ready RFCs in time for the next RFC ballot.

RFC Cover Sheet Information Recorded At This Stage:

Identification (with associated dates) of joint-interest committee participation in the technical coordination process.

Date the summary report was made to the IGES Project Committee.

RFC Actions Taken at This Stage:

1. See items 5,6 , and 7 , Section 3.2.3.

2. The Change Control Secretary supplies the IGES Ballot Coordinator with the ballot-ready copy of all RFC Forms that have been given final approval for ballot. As a means of doublechecking the contents of the upcoming ballot, the Secretary also mails a copy of the RFC Forms to the IGES Editor and the IGES Project Manager.

\subsubsection{The RFC Ballot}

The RFC (mail) ballot is the process by which voting members of the IPO General Assembly can comment on and vote approval or disapproval of one or more RFCs. Normally, RFC ballot periods span successive IPO meetings, so there is an alternating cycle of mail ballots and IPO meetings.

RFCs that have been given approval for ballot through oversight review by the IGES Project Committee are eligible for RFC ballot. Eligible RFCs are automatically included in the next RFC ballot.

The RFC Form portion of each RFC is what is actually included in the RFC ballot.

For each RFC ballot conducted, the IGES Ballot Coordinator assembles and mails a ballot package to each member of the Ballot Group. Each ballot recipient should return the ballot by the stated deadline.

The IGES Ballot Group concept is intended to avoid the cost of wasted mailings to the 90 percent of IPO members who do not vote. Any voting member of the IPO may become a member of the Ballot Group by request, and will remain in the group until the member requests removal. All IPO members who become qualified to vote will be mailed one ballot that includes a "request form" to become a member of the Ballot Group. Members who do not vote or return the request form will 
not receive future ballots. However, if they want to resume voting in the future, they may do so by sending a written request to the IGES Ballot Coordinator, who will add them to the Ballot Group. An RFC ballot form shall be provided by the IGES Ballot Coordinator. This form identifies the RFCs being balloted and records the vote registered. Voting choices are: Approve, Approve With Comment, Disapprove (comment giving reasons is required), and Abstain.

Ballot recipients returning a ballot cast their vote ${ }^{5}$ on each RFC. If a returned ballot does not indicate a choice of Approve, Approve With Comment, or Disapprove for a particular RFC, the vote for that RFC is interpreted as "Abstain."

As described in Section 3.3.4, an RFC may possibly be balloted more than once as a result of technical modifications arising from comments; in such cases, the RFC version is incremented.

\subsubsection{Ballot Summary and Ballot Evaluation}

After an RFC has been included in the mail ballot, it is assigned "Balloted" status. RFC ballot results are summarized and evaluated for each RFC.

A Ballot Summary Report is prepared by the IGES Ballot Coordinator following each RFC ballot. The Ballot Summary Report contains:

identification of the RFC ballot being summarized, including the beginning and end dates of the ballot period,

the number of ballot packages sent out in each membership category (Vendor, User, General Interest), and the total number sent out,

the list of ballot recipients,

40 the number of ballot packages unreturned in each membership category, and the total number unreturned,

20 the list of ballot recipients returning a ballot,

the RFC number, issue revision, and Title of each RFC balloted, along with the vote tally for each RFC,

a report of all comments received, indicating for each comment the name, address and telephone number of the commentor, and the voting preference cast by the commentor. Comments will be transcribed to the Comment Disposition Form that will be delivered to the custodian technical committee's chairperson for use in processing the comment.

The Ballot Summary Report is the basis for evaluation of the ballot by the IGES Project Committee. The purpose of the ballot evaluation is to officially review, accept and report the results of the RFC ballot and to possibly issue statements (comments and/or recommendations) concerning the RFCs to the appropriate custodian committees. The report of the review and acceptance, along with any statements, form the primary contents of the Ballot Evaluation Report.

An RFC receiving only "Approve" or "Abstain" votes is automatically assigned "ECO" status by the IGES Project Committee; otherwise, it is returned with "Balloted" status to the custodian committee for resolution of disapprovals and comments.

5. If the voter has several approval comments concerning editorial issues such as misspellings, one option is to mark up a copy of the RFC using a contrasting color and mail it with your ballot. NOTE: Do not use this method for technical disapproval comments. Do not fax this reply since the color is lost, making reading difficult. 
The Ballot Evaluation Report will take the form of a cover letter to the Ballot Summary Report. It will also report on the manner in which consensus of the IGES Project Committee was achieved, e.g., at a meeting of the Committee, by a conference call, by e-mail, etc.

RFC Actions Taken At This Stage:

1. The Project Manager performs the necessary coordination for preparation of the Ballot Evaluation Report.

2. The Ballot Evaluation Report is distributed to IGES Project Committee members, authors of RFCs on the ballot, and ballot respondents. Comment Disposition Forms containing the transcribed comments are distributed to custodian committee chairpersons in time to allow scheduling them as agenda items at the next IPO meeting.

3. If an RFC receives either approval or disapproval comments, the RFC author should attempt to contact each commentor personally before the meeting in an attempt to resolve each comment; this avoids problems if the commentor does not attend the next IPO meeting. Any comments resolutions handled by the RFC author shall be communicated to the custodian committee chairperson for entry on the Comment Disposition Forms.

\subsubsection{Ballot Follow-On Work in Custodian Committees}

RFCs that receive only "Approve" and "Abstain" votes in the ballot process are approved and move directly to "ECO" status without return to the custodian committee.

RFCs receiving approval or disapproval comments in the ballot process are returned to the custodian committee in the "Balloted" state, and additional work will be required to resolve all comments.

Ordinarily, "Approval" comments identify typographical errors or lack of clarity, and "Disapproval" comments identify technical problems. Some commentors do not always make this distinction; therefore, all comments are classified as "technical" or "editorial." Both technical and editorial comments are further classified as "persuasive" or "non-persuasive."

All persuasive comments must be resolved by amending the RFC; the custodian committee is encouraged to consider any suggestions from the IGES Project Committee in the Ballot Evaluation Report. If resolving the comments results in any technical changes, the custodian committee will vote to determine if they change the intent or functionality of the RFC. If intent or functionality changes, the custodian committee shall return the amended RFC to the IGES Project Committee with "Recommended for Ballot" status. Otherwise, the custodian committee will return the amended RFC to the IGES Project Committee with "Recommended for Gray Pages" or "Recommended for Update" status depending on whether Gray Page testing is required, has been completed, or is not required.

In the event persuasive technical comments indicate the RFC cannot be salvaged, the custodian committee will encourage the author to withdraw it, or as a last resort, vote to cancel it. Any RFC author not accepting the "Cancelled" status may appeal it to the IGES Project Committee. The RFC is returned to the IGES Project Committee with either "Withdrawn" or "Cancelled" status. 
Figure 4 illustrates the ballot outcomes for an RFC.

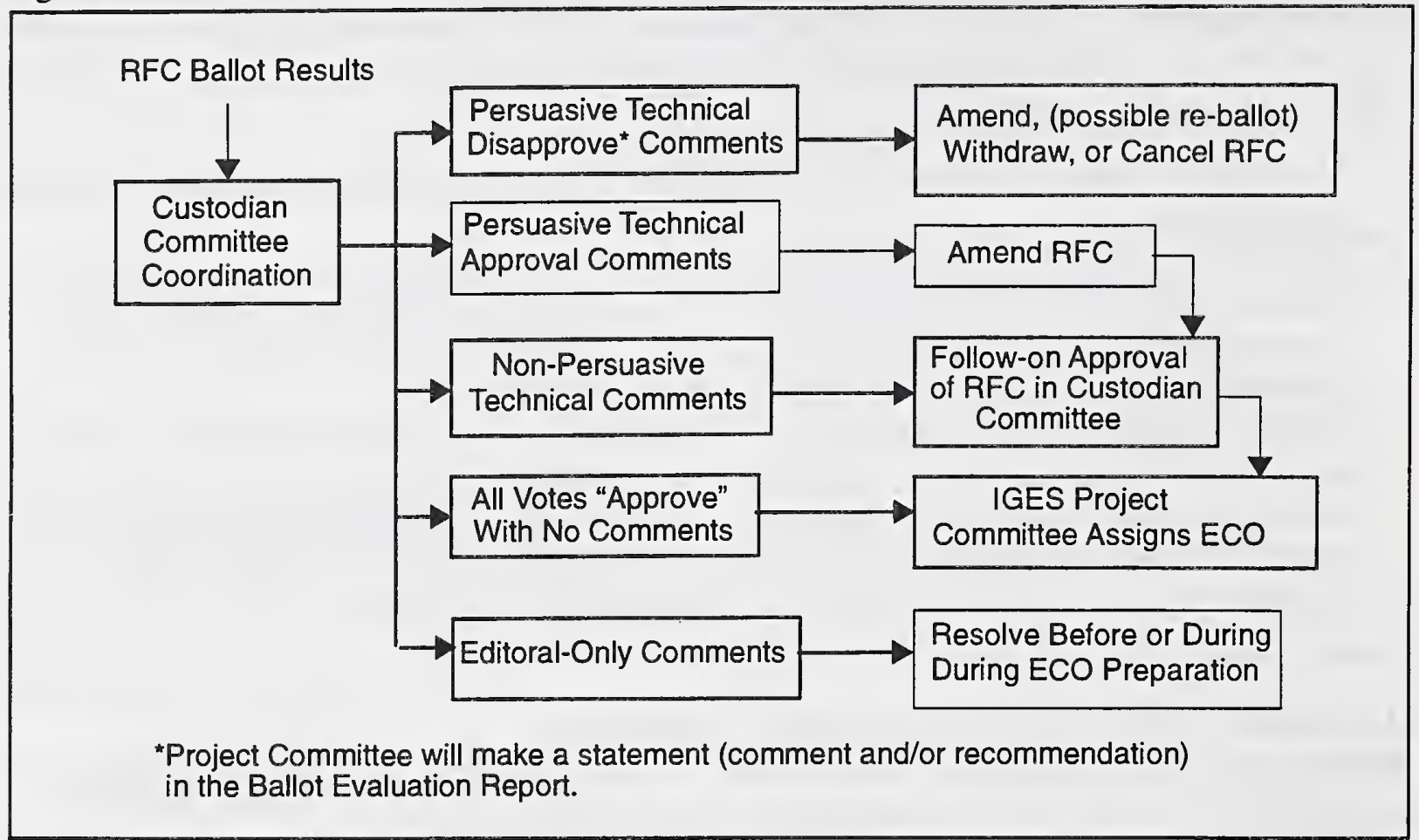

Figure 4. RFC Ballot Results

\section{Announcement Of Ballot Outcomes}

Individual comments on an RFC ballot are separated into distinct issues on the Comment Disposition Form.

The ballot outcome for each RFC is summarized in the minutes of the custodian committee.

\section{Resolution Of Individual Comments}

The requirements concerning resolution of individual comments are:

1. All comments received must be addressed.

2. A concerted effort must be made to resolve each comment.

3. All commentors shall be advised in writing of comment disposition unless they attend the technical meeting when the comment is addressed. The simplest method of providing this written disposition is to photocopy the completed Comment Disposition Form and deliver it to the commentor.

Adequate records must be maintained to provide evidence of compliance with these requirements. Resolution of individual ballot comments follows the process outlined in Figure 5. 


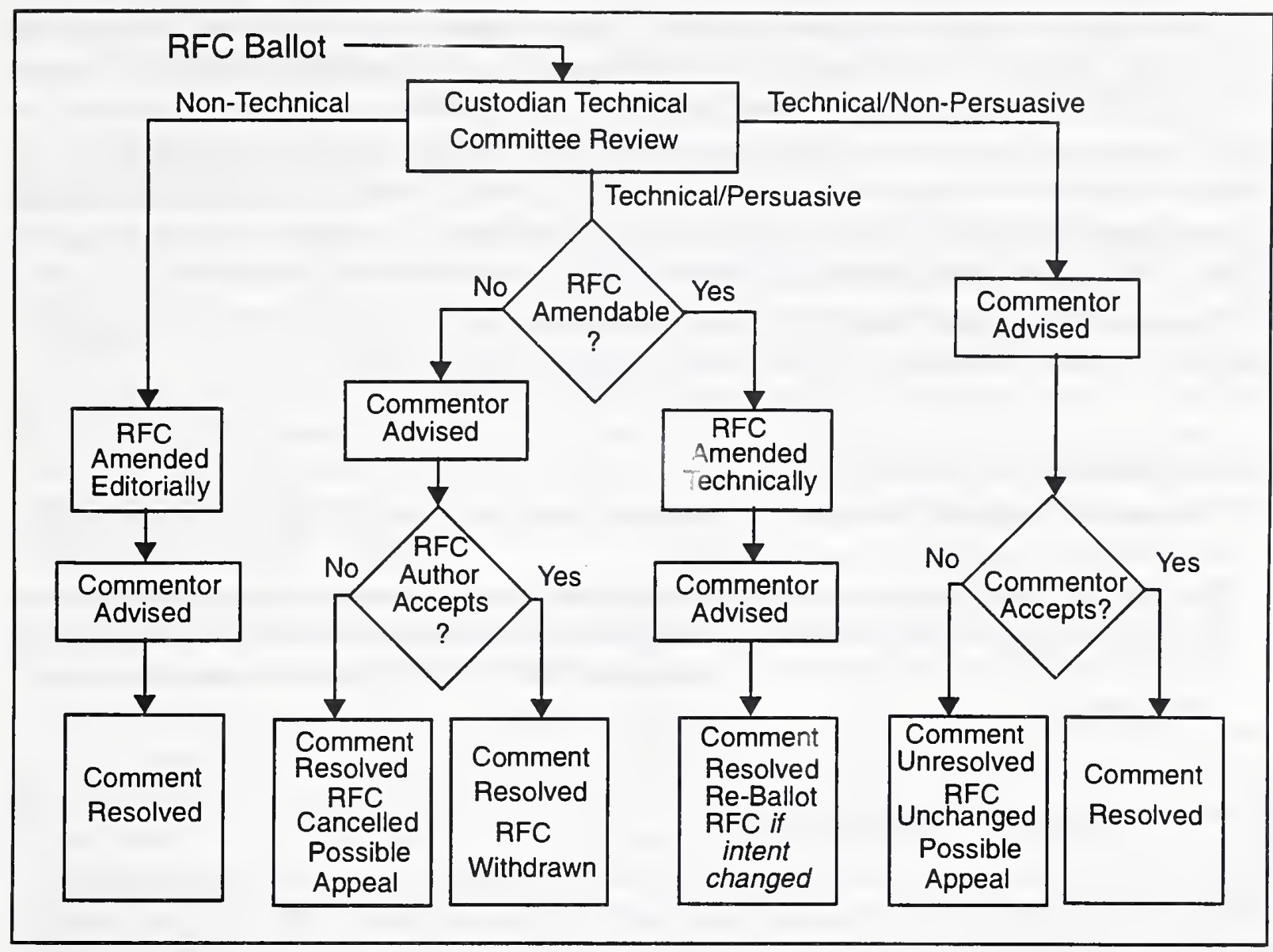

Figure 5. RFC Comment Resolution in Custodian Committee

Comments will be organized by type and classification: the types are editorial and technical; the classes are persuasive and non-persuasive.

The operating procedures used by custodian committees to ensure that the above requirements are met when this process is used are:

Custodian committee responsibilities for each comment are traceable to a specific individual who is identified in the minutes of the custodian committee. In most cases, this will be the author of the RFC. In any case, it is the responsibility of the chairperson of the custodian committee to see that such an individual is identified. Responsibilities for each comment include:

- $\quad$ Communicating as necessary with the commentor concerning the comment and passing along the substance of this communication to the custodian committee. In many cases, personal communication with the commentor prior to the IPO or Project meeting can identify an acceptable resolution of the comment (i.e., the commentor compromises by agreeing to withdraw the comment if a specified change is made).

- Ensuring that the commentor is informed in writing of the categorization of the comment by the custodian committee and the action taken upon it. (This notification is not required if commentor attends the technical committee meeting because the commentor will 
receive the committee's minutes.)

The actions taken can include amending the RFC and recommending it for "Ballot," "Gray Page Testing," or "Update" (depending on the nature of amendment); requesting the author withdraw a non-amendable RFC; or voting to "Cancel" a non-amendable RFC.

If RFC authors or commentors disagree with custodian committee actions, and reasonable attempts to resolve the disagreement at the custodian committee level fail, the author or commentor may appeal to the IGES Project Committee. The IGES Project Committee's decision regarding appeals shall appear in the minutes. See Section 5.0 for more information on appeals.

The Comment Disposition Form(s) documenting the comment resolution process are returned to the Change Control Secretary by the custodian committee chairperson.

\section{RFC Actions Taken At This Stage:}

1. For each RFC balloted, the custodian committee chairperson ensures that the committee minutes contain the ballot outcome for the RFC.

2. For each RFC balloted, the custodian committee chairperson ensures that the operating procedures for resolution of individual ballot comments (procedures \#1 and \#2 above) are followed.

3. The custodian committee chairperson notifies the IGES Project Manager of any impending appeal situation.

\subsection{The Gray Page Testing Process}

Version 4.0 and later versions of The Initial Graphics Exchange Specification, as well as ANSI ASME Y14.26M-1989 (Digital Representation For The Communication of Product Data, which is based on IGES 4.0), contain an Untested Entities Appendix, also referred to as the "Gray Pages." This "Gray Page" technique has been identified as causing extra editorial work and increased potential for errors when entities are moved into the main body of the Specification; for this reason, after Version 5.2, untested entities will appear in their "final" place in the main body of the Specification, but will be prefixed by an UNTESTED designation. In these procedures, the term "untested entity" designates an entity that has not completed Gray Page testing, regardless of whether it appears in the Untested Entities Appendix or in the main body with an UNTESTED designation. Implementors are encouraged to attempt implementation of untested entities, and are requested to inform the IGES Project Committee if it becomes apparent that changes to them are required.

Untested entities generally extend the capability of the Specification. These entities have been approved in RFC ballot and have been tentatively approved within their custodian committee, but are required to successfully undergo certain testing before they can be fully approved and be assigned "Recommended for Update" status by the custodian committee, and eventually become part of the main body of the Specification. This testing is the "Gray Page" testing referred to in Section 2.2.

The procedures in this section are intended to assure that untested entities are adequately tested and results reviewed before being assigned "Recommended for Update" status. Also, procedures are given for reviewing the results of Gray Page testing by the Gray Page Committee. 


\subsubsection{The Gray Pages}

All proposed changes to the Specification (i.e., all RFCs) are divided into two groups, those that require Gray Page testing and those that do not. Generally speaking, RFCs requiring this testing are extensions, while those not requiring the testing are clarifications or fixes. See Section 2.2. The IGES Project Committee makes the decision concerning the group to which a new RFC is to belong. See Sections 3.2.2 and 3.2.3.

After an RFC that requires Gray Page testing has been assigned "Recommended for Gray Pages" status within the custodian committee, it is presented to the IGES Project Committee for oversight review. See Sections 3.2.3 and 3.4.3. If oversight review results in approval, the IGES Project Committee will assign "ECO" status to the RFC with a direction to the IGES Editor to designate the entity as UNTESTED. If Gray Page Testing is completed prior to publication of the next version of the Specification, the IGES Project Committee may assign "ECO" status again with a direction to the IGES Editor to remove the entity's UNTESTED designation; otherwise, it will remain an UNTESTED entity until testing is complete and the custodian committee assigns "Recommended for Update" status.

Successful early completion of Gray Page testing will allow the custodian committee to assign "Recommended for Update" status before oversight review by the IGES Project Committee, thereby avoiding the entity's UNTESTED designation.

Gray Page testing for an RFC consists of demonstrating the use of three processor implementations to successfully pass information between three systems. The information passed shall be the same information as that included in the RFC. It is understood that the testing requirements will depend greatly on the nature of the RFC. For example, the involvement of a system capable only of visual display might be appropriate in some cases, while a system capable of making internal modifications of the information being transferred would be required in other cases. The primary purpose of Gray Page testing is to prove that new entities are technically correct and can be implemented to exchange the intended information, not to demonstrate marketplace demand. Removing an entity's UNTESTED designation finalizes it, thereby encouraging more implementations.

For each new RFC requiring Gray Page testing, a Gray Page Test Plan for demonstrating the successful passing of information is to be part of the RFC before it is assigned "Approved for Ballot" status by the IGES Project Committee. Origination of the Test Plan, coordination of it with the Gray Page Committee, and finalization of it are the responsibility of the custodian committee for the RFC. The Test Plan is addressed when the "Recommended for Gray Pages" status is reported to the IGES Project Committee by the custodian committee's chairperson. See Section 3.3.1. The Test Plan is balloted and commented upon in the RFC ballot along with the RFC Form itself. Comments on the Test Plan will be handled as any comment on the RFC. See Section 3.3.4.

Coordination with the Gray Page Committee for review of the Test Plan will be initiated when comments from the ballot process have been resolved and the Plan is able to be recommended by the custodian committee. The Gray Page Committee will review the Test Plan and make comments. A Gray Page Test Plan Review letter will be issued back to the custodian committee and used in finalizing the Test Plan.

Within the custodian committee, attempts will likely be made to delegate responsibility for the Test Plan to the author of the RFC. The idea here is to encourage the person or persons most interested in the RFC to see that Gray Page testing is addressed in a timely manner, with the optimum result being that the entity never receives an UNTESTED designation 
The Test Plan is to describe the information to be passed and the details of the test, i.e., what constitutes successful exchange of the information. The Test Plan will be structured to consist of one or more test cases.

Ideally, the Test Plan will identify the individual or individuals from whom commitments have been obtained for constructing the test cases and carrying out the testing. If feasible, the Test Plan will include the names of the systems to be used in the test. Based on the commitments obtained, the Test Plan will include a schedule to allow the IGES Project Committee to judge progress towards its completion.

\section{Custodian Committee Responsibilities For Gray Page Testing:}

For each RFC requiring Gray Page testing:

1. Originate a proposed Test Plan for inclusion along with the RFC Form in the RFC ballot.

2. Prepare a recommended Test Plan taking into account the results of the RFC ballot, present this to the Gray Page Committee for review, and act on the results of the review to finalize the Plan.

3. Obtain commitment to carry out the Test Plan, and prepare a schedule of anticipated activities accordingly.

4. Report to the IGES Project Committee concerning approval of the RFC for Gray Page testing.

5. Track the testing activity as it progresses.

6. Arrange for the Gray Page Committee to review the test results against the finalized Test Plan.

7. Receive the Gray Page Testing Review letter (see below) from the Gray Page Committee.

8. Archive the Test Plan and individual test case material as necessary in the RFC Folder.

9. Report to the IGES Project Committee concerning approval of the RFC for ECO.

\subsubsection{The Gray Page Committee}

Following its review of the test results, the Gray Page Committee will issue a Gray Page Testing Review letter. This committee is a subcommittee of the IGES Project Committee (not a Technical Committee of the IPO General Assembly).

The review of the Gray Page test results will focus on the adherence of the testing activities to the Test Plan, on the completeness with respect to the test cases listed in the Test Plan, and on the quality of the individual test case results.

Following its review of the test results, the Gray Page Committee will issue a second Gray Page Test Review letter for the RFC (as in Section 3.4.1; the first concerned the review of the final Test Plan). This letter will make a recommendation as to whether or not the testing for the RFC was extensive enough and successful enough to support removing an entity's UNTESTED designation.

\section{Gray Page Committee Responsibilities For Gray Page Testing:}

1. Review each recommended Test Plan and issue a Gray Page Test Plan Review letter.

2. Review Gray Page test results for each RFC requiring Gray Page testing and issue a Gray Page Testing Review letter to the IGES Project Committee recommending whether or not the 
testing is sufficient to remove an entity's UNTESTED designation.

3. The Head of the Gray Page Committee will report regularly to the IGES Project Committee concerning its activities.

\subsubsection{Oversight Review for Gray Page Testing Approval}

The IGES Project Committee performs oversight review for Gray Page testing approval on each RFC that has reached "Recommended For Gray Pages" status within the custodian committee. As given in Section 3.2.3, the oversight review is initiated by the report of the custodian committee chairperson to the IGES Project Committee.

The report will contain at least the following:

RFC ballot results, ballot comments, and their resolution, especially concerning the proposed Test Plan.

Actions taken to finalize the Test Plan as a result of the Gray Page Test Plan Review letter

Commitment and anticipated schedule for carrying out the Test Plan.

Options for the IGES Project Committee following oversight review were given in Section 3.2.3.

\section{$\underline{\text { RFC Cover Sheet Information Recorded At This Stage: }}$}

See items 4 and 5, Section 3.2.3.

RFC Actions Taken At This Stage:

See items 5, 6, and 7, Section 3.2.3.

\subsubsection{Existing Gray Page Material Recommendations}

For these entities, responsibility for finalizing the Gray Page Test Plan lies with the custodian committee for the original RFC. However, the Gray Page Committee will assist by working actively to originate a Test Plan for each entity to be tested that will be presented to the custodian committee for finalization. The Gray Page Committee will seek to identify parties willing to participate in the testing of an entity, and will work with those parties in the origination of the Test Plan. The Test Plan will be presented to the custodian committee for finalization along with a Gray Page Test Plan Review letter addressing any relevant issues. Other procedures remain as in Section 3.4.3 for new RFCs.

In view of the large number of entities currently designated as UNTESTED, the Gray Page Committee is encouraged to recruit help in this area.

When it becomes necessary to set priorities as to which entities to address first, the Gray Page Committee is encouraged to take direction from the IGES Project Committee.

\subsection{The Edit Change Order (ECO) Process}

The Edit Change Order (ECO) is the mechanism by which the IGES Editor is officially informed of the content of a change to the Specification, and also authorized to make the change to the mas- 
ter copy of the Specification.

Typically, an ECO represents the culmination of work on an RFC that encompasses technical coordination, balloting, resolution of comments, and achievement of final technical and editorial consensus. In these cases, the ECO is essentially the RFC Form as it existed when the RFC reached "Recommended for Gray Pages" or "Recommended For Update" status in the custodian committee. In other cases, an ECO originates within the IGES Project Committee itself and concerns editorial changes to the Specification

ECOs are proposed to and approved by the IGES Project Committee. An ECO may be proposed to the IGES Project Committee by either a chairperson of a custodian committee for an RFC, by the Chairperson of the Gray Page Committee, or by a voting member of the IGES Project Committee on behalf of the entire IGES Project Committee. In particular, the IGES Editor can propose an $\mathrm{ECO}$ on behalf of the entire committee.

\subsubsection{The Makeup of an ECO}

An ECO consists of an RFC Cover Sheet together with the content of the change being authorized to the Specification.

The change content is presented in its "integrated form," i.e., all parts of the Specification affected by the change are accurately depicted as they will appear after the change has been made. Temporary entity type and form numbers have been replaced by actual numbers. Logically complete portions of the Specification are depicted.

Since each RFC is balloted in its integrated form, the ECO is equivalent to the proposed change portion of the RFC; however, editorial changes made after balloting will require manual identification and Editor notifying.

The Cover Sheet contains the necessary administrative information for the ECO. See Appendix B for an example of the RFC Cover Sheet.

ECOs are numbered in increasing order, although not necessarily sequentially. Sequential "issue" versions ("A," "B," "C," etc.) of an ECO can exist; for example, RFCs assigned "Recommended for Gray Pages" status will result in an ECO to direct the IGES Editor to insert the entity with an UNTESTED designation. If testing is completed successfully before the IGES Editor takes action on the ECO, a new version may be issued to remove the UNTESTED designation; however, usually a new ECO number will be created.

All ECOs exist officially in both paper and electronic form. They are archived by the Change Control Secretary in their paper form. All issue versions are archived. In their electronic form, they are accepted and managed as electronic files by the IGES Editor for integration into the Specification.

\subsubsection{Oversight Review for ECO Approval}

The IGES Project Committee performs oversight review on each proposed ECO. It is presumed that the change content of each proposed ECO exists in both paper and electronic form.

As given in Section 3.2.3, when the proposed ECO is for an RFC that has reached "Recom- 
mended for Gray Pages" or "Recommended for Update" status in custodian committee, the oversight review is initiated by a report containing at least the following:

1. RFC ballot results, and ballot comments and their resolution.

2. Gray Page testing (if it was required).

3. The state of the consensus agreement between the custodian committee and the IGES Editor concerning the change content of the ECO.

Options for the IGES Project Committee following oversight review were given in Section 3.2.3.

If the oversight review results in an ECO approved or conditionally approved, the "Approval For Release" signatures on the RFC Cover Sheet must be obtained from the presenter of the ECO and from the Project Manager; the cover sheet and a final form of the ECO will be supplied to the IGES Editor. For approved ECOs, the signatures can be obtained immediately, the Cover Sheet can be attached to the RFC as presented, and these can be turned over to the IGES Editor. For ECOs approved conditionally, the necessary changes should be minor enough that the presenter of the RFC can sign the RFC Cover Sheet and attach it to a marked-up copy of the RFC presented for oversight review. The signature of the Project Manager is added later after the editorial changes have been made, and this signature denotes that the RFC is in final form and is ready to be supplied to the IGES Editor. In rare cases, editorial changes resulting from oversight review may be extensive enough to warrant generation of an updated version of the ECO before the Cover Sheet can be signed.

When the proposed ECO is being presented by a voting member of the IGES Project Committee on behalf of the entire Committee, a report will be made summarizing the reasoning and justifying the editorial change. This type of ECO ordinarily will be initiated by the IGES Editor to fix grammar or typographical errors of a non-technical nature. The IGES Project Committee will either accept or reject the proposed ECO and record this in the minutes.

Consideration and approval of ECOs constitutes official business of the IGES Project Committee.

\section{RFC Cover Sheet:}

1. The presenter of the RFC fills out and signs the RFC Cover Sheet and supplies the Project Manager with the paper copy of the RFC.

2. The Project Manager signs the RFC Cover Sheet.

\section{ECO Actions Taken:}

1. The Project Manager passes the ECO to the Change Control Secretary for archiving, supplies the Editor with a paper copy of the ECO, and verifies that the Editor has an electronic copy of the integrated form of the RFC.

2. The Change Control Secretary archives a paper copy of the ECO.

3. The Editor manages the electronic form of the ECO. 


\subsection{The Process for Approving Major Changes to the Specifi- cation}

\subsection{New Versions of the Specification}

1. The IGES Project Committee will consider a motion to approve a new version of the Specification. Any member of the Committee may initiate this motion, but the IGES Editor is most likely to do so following a "reading session" by members of the IGES Editorial Committee and the IGES Project Committee. The initiator will prepare an RFC for tracking purposes and will obtain a number from the Change Control Secretary.

2. If the motion passes, the IGES Project Committee will assign "Approved for Ballot" immediately. The IGES Project Committee is the custodian committee for this RFC, and all Technical Committees of the IPO are considered "joint-interest" committees. The RFC will not require Gray Page Testing.

3. A draft of the proposed new version of the Specification will be prepared and distributed at the next meeting of the IPO to minimize mailing costs. The "draft" status will be marked clearly. An announcement will be made to remind members to complete a ballot request form if they currently are not a member of the Ballot Group and wish to vote.

4. Members of the Ballot Group will receive a mail ballot that concerns only the RFC initiated in step 2 concerning the question of approving the new version of the Specification. (No other RFCs may be included with this ballot.) This ballot also will include instructions for receiving a copy of the proposed new version of the Specification if one was not obtained at the IPO meeting.

5. The mail ballot is handled according to procedures in Section 3.3.2; the response deadline shall allow a minimum of two months for review.

6. Comments on the mail ballot will be handled by the IGES Project Committee or distributed to the most qualified joint-interest Technical Committee for handling according to procedures in Section 3.3.4. Due to prior review of technical changes in the RFC process, it is assumed that: 1) most comments will be editorial, and 2) all comments will be resolvable. (Step 8 below deals with comment resolution requiring reballot.) Technical comments concerning areas of the Specification that did not change from the prior version, or any comments duplicating previously classified "non-persuasive" comments are inappropriate and will be classified "nonpersuasive."

7. If resolving comments requires making changes, each joint-interest committee will contact the IGES Editor to amend the master copy of the proposed new Specification, and return the tracking RFC to the IGES Project Committee with the "Approved for Update" status assigned. If this is impossible, the chairperson of the joint-interest committee should obtain assistance from the IGES Project Committee to resolve the comment causing the difficulty. In the event that persuasive/technical comment(s) cause the need to reballot, a new version of the tracking RFC will be balloted, but it will refer only to the specific areas needing reballoting - the entire Specification will not be reballoted. Any comments on other subjects are inappropriate and will be classified "non-persuasive."

8. Following receipt of "Approved for Update" status from all joint-interest committees, the IGES Project will perform oversight review of the comment dispositions; in most cases, this will be at an interim meeting due to the time required. Following successful review, the 
IGES Project Committee will issue an ECO to the IGES Editor directing the release of the new version of the Specification. The new version of the Specification will be submitted to ANSI as a proposed American National Standard according to procedures in the IGES/PDES Organization Reference Manual (available from the IPO Office or at IPO meetings).

The minutes of the IGES Project Committee and of all the joint-interest Technical Committees, and all the required paperwork connected with approval, balloting, and comment resolutici if the tracking RFC initiated above, constitute official business of the IGE/PDES Organization.

\subsection{Application Protocols}

In addition to IGES itself, this document also pertains to the related "Application Protocol" additions. The following apply:

1. The IGES Project Committee will consider a motion from the chairperson of the application protocol's technical committee to approve an application protocol. The initiator will prepare an RFC for tracking purposes and will obtain a number from the Change Control Secretary.

2. If the motion passes, the IGES Project Committee will assign "Approved for Ballot" immediately. The application protocol's technical committee is the custodian committee for this RFC. The RFC will not require Gray Page testing.

3. A draft of the proposed application protocol will be prepared and distributed at the next meeting of the IPO to minimize mailing costs. The "draft" status will be marked clearly. An announcement will be made to remind members to complete a ballot request form if they currently are not a member of the Ballot Group and wish to vote.

4. Members of the Ballot Group will receive a mail ballot concerning the question of approving the Application Protocol (other RFCs may be included with this ballot). This ballot also will include instructions for receiving a copy of the proposed application protocol if one was not obtained at the IPO meeting.

5. The mail ballot is handled according to procedures in Section 3.3.2; the response deadline shall allow a minimum of one month for review.

6. Comments on the mail ballot will be handled according to procedures in Section 3.3.4; due to prior review of any technical changes needed by the application protocol in the RFC process, it is assumed that: 1) most comments will be editorial, and 2) all comments will be resolvable.

7. If resolving comments requires making changes, the application protocol's technical committee will amend the master copy of the proposed application protocol and return the tracking RFC to the IGES Project Committee with the "Approved for Update" status assigned. If this is impossible, the chairperson of the joint-interest committee should obtain assistance from the IGES Project Committee to resolve the comment causing the difficulty. In the event that persuasive, technical comment(s) cause the need to reballot, a new version of the tracking RFC will be balloted, but it will refer only to the specific areas needing reballoting-the entire application protocol will not be reballoted. Any comments on other subjects are inappropriate and will be classified "non-persuasive."

8. Following receipt of "Approved for Update" status from the application protocol's technical committee, the IGES Project will perform oversight review of the comment disposition. Following successful review, the IGES Project Committee will issue an ECO to the IGES Editor 
directing the release of the application protocol. The minutes of the IGES Project Committee and of the application protocol's technical committee, and all the required paperwork connected with approval, balloting, and comment resolution of the tracking RFC initiated above, constitute official business of the IGES/PDES Organization.

\subsection{The Appeals Process}

The process of maintaining and changing the Initial Graphics Exchange Specification is based upon the concept of "consensus" (i.e., substantial agreement among most of those concerned; see section 1.1). These procedures assure that

- $\quad$ serious proposals to change the Specification, as well as any comments concerning such proposals, will be given the most careful consideration possible by at least two groups of qualified persons (i.e., the custodian technical committee, and the IGES Project Committee), and

- the results of this consideration will be documented completely and communicated unambiguously to those involved.

In most cases, informal resolution of disagreements in the technical committee meeting are the most effective and efficient way to solve problems. Ideally, consensus will be reached via compromise followed by unanimous agreement; however, compromise can be impossible in technical issues having mutually exclusive alternatives. When this happens, the committee's Chairperson ordinarily puts the matter to a vote, and exercises discretion to determine if the vote is sufficiently one-sided to qualify as "consensus" (i.e., a simple majority is not enough); if it is, the opinion of the majority shall prevail. In spite of this, anyone holding the minority opinion who is or will be materially and adversely affected by the outcome has the right to pursue the formal process described below to appeal the decision.

\subsection{Definition of Appellants}

1. Any RFC author whose RFC is assigned "Cancelled" status by the custodian technical committee of the IPO has the right of appeal to the IGES Project Committee if the matter has been discussed and cannot be resolved within the custodian technical committee meeting.

2. All commentors participating in the RFC ballot process who disagree with the classification of their comment(s) as "non-persuasive" have the right of appeal to the IGES Project Committee if the matter has been discussed and cannot be resolved within the custodian Technical Committee meeting.

\subsection{Appeals procedure}

To appeal, send photocopies of original comment(s) and the IGES RFC Comment Disposition Form (if received) to the IGES Project Manager with a signed statement indicating the area of disagreement and the outcome that would satisfy the appellant's concerns. Appellants will be scheduled on the agenda of the next IGES Project Committee meeting to discuss the situation; after discussion, the Committee will propose and vote on action to resolve the matter. Appellants who 
continue to feel they are adversely and materially affected may appeal to the IPO Chairperson for binding arbitration. Refer to the IGES/PDES Organization Reference Manual (re-published for each IPO meeting) for more information.

\subsection{IGES Change Tracking}

Presently, the IGES Change Control Secretary manages IGES Change Tracking. RFC status at any point in time is available from a database containing the electronic master of the RFC cover sheets. In addition, a summary "tracking worksheet" can be produced as part of the process of managing the RFC cover sheets. The electronic cover sheet file is available on a computer at the IPO meetings so Technical Committee Chairpersons can make updates if desired. (The process is simple enough that even those who are not trained in the hypertext application used can succeed.) There is also a procedural diagram available interactively. Copies of some of these screens appear in a companion document referenced in Preface footnote 1.

In the future, it is hoped that the entire RFC process will be managed using a database, thereby increasing both the ability to share information and the ease of keeping it up to date. For now, many people who are participants in this process have access to electronic mail. In particular, anything sent via e-mail to the IGES Change Control Secretary can be automatically "exploded" to a mailing list for wider distribution. The Internet World Wide Web (see Preface for URLs) is used to provide the Project with a list of the active RFCs and the ECOs as they are approved for the next IGES version.

Information in this section will be expanded as procedures connected with IGES Change are refined during the application of other procedures in this document.

\subsection{Informal Forums for IGES Issues, Analyses, etc.}

As discussed in prior sections of this document, potential RFC authors who identify a problem to be solved are expected to also identify several proposed alternatives to solve it either by themselves or by consultation with technical experts in the area of concern. In the event that at least one apparently useful solution cannot be identified, the formal RFC process should not be initiated; the potential RFC author should pursue the following suggestions first:

Check the IGES Recommended Practices Guide to see if the problem situation is addressed. In many instances, a Recommended Practice is established prior to or concurrently with an in-process RFC (this happens because Proposed Recommended Practices can be put in place very quickly compared to the formal RFC process). In addition, such checking may indicate a Proposed Recommended Practice is a more appropriate way to deal with the situation involved, and authoring one is much easier than an RFC. Furthermore, the possibility of quick approval of a Recommended Practice means a policy to solve a problem can be in place sooner, thereby minimizing continued harm from the problem as well as quantifying the value of a formal RFC solution. Consult the Chairperson of the IGES Implementors Committee for more information. 
Attend a meeting of the Technical Committee of the IPO whose area of expertise seems appropriate to address the area of concern (e.g., discuss a mathematics issue with the Geometry Committee). If unsure of which committee is most appropriate, start with the Implementors' Committee due to their familiarity with the software systems which actually perform the exchange processing. At the meeting, someone is bound to offer information that will help in making a decision to write an RFC or drop the matter. If not, it is likely that an ad hoc subcommittee will be formed in an attempt to deal with the problem. In the past, this method has been the most successful in dealing promptly with problems that have no apparent solution. Afterward, the results obtained should indicate if the next action should be a Proposed Recommended Practice, an RFC, or both at once.

Ask to speak at the plenary session of the IPO General Assembly. Again, someone is likely to offer suggestions either immediately or afterwards. If reluctant to speak publicly, request the IGES Project Manager to bring up the issue for you or submit an anonymous request.

Write a paper and distribute it for comments. In the past, the RFC process included a "Change Analysis" paper that identified a problem, but not always its solution. Efficiency considerations dictated dropping this step as a formal requirement because most authors were found to have a solution in mind and could therefore go straight to the RFC-authorship step. Nothing in this procedure prohibits this type of paper; in fact, this method is preferred to the idea of authoring an RFC with a useless solution in an attempt to get feedback. Such papers may be distributed at the IPO meetings. Their availability can be announced in the daily newsletter by requesting this at the IPO meeting office. Other distribution options include sending the paper out with specific Technical Committee meeting minutes (consult Chairperson of the relevant Technical Committee), or requesting the paper be included in the mail ballot to the Ballot Group (consult the IGES Project Manager).

Pursuing the above suggestions will save both time and unnecessary effort for everyone involved in the RFC process. Past experience convincingly demonstrates that RFCs containing incomplete or ineffective solutions to problems have consumed tremendous resources by their repeated iterations through the process. Spending extra effort initially on informal research will pay future dividends in the form of reduced rework, decreased processing time, and increased acceptance.

Everyone will recognize and appreciate an effort to prepare complete, high-quality work.

\subsection{Step-by-Step User Guide to the RFC Process}

This section documents the entire RFC Process in a straightforward, step-by-step manner. These steps are consistent with the detailed description of the process appearing in prior sections. The necessary simplification may not cover all details, particularly if problems occur with a specific RFC-in this case, refer to prior applicable sections for more information. The steps are written in a short, direct style and are organized into a checklist that may be especially useful to new RFC authors.

Section 8.1 covers processing of "normal" priority RFCs; i.e., RFCs submitted more than six 
months prior to the anticipated publication date of the next version of the Specification. RFCs in this category may cover any appropriate subject because there is ample time for consideration and work to resolve any problems. Potential authors should be aware that controversial or complicated RFCs have required up to two years for completion of processing.

Section 8.2 covers processing of "expedited" priority RFCs; i.e., RFCs submitted less than six months prior to the anticipated publication date of the next version of the Specification. Expediting refers to getting the RFC from concept to ballot quickly. It is very difficult to speed up the subsequent ballot and comments resolution process, but those processes also are likely to be faster if the limitations below are observed. RFCs in this category are limited in subject and content due to the limited time frame. In general, these limitations apply:

- The author has researched the problem prior to the meeting to identify both solutions and support from those who may be affected, and has prepared this information in paper form to facilitate immediate committee discussion.

- The Technical Committee to be assigned as custodian should be obvious; there should be no joint-interest committee involvement.

- $\quad$ Both the problem and its proposed solution should be clear, obvious, and non-controversial (e.g., adding a new property is a good candidate; changing a complex entity like the Trimmed Surface is not).

- The entire process should be started early in the week at an IPO meeting so that the electronic master of the Specification, the designated document and IGES processors for the Specification, the IGES Editorial Committee, and the IGES Change Control Secretary all are available to enable production of the "ballot-ready" RFC.

Although there are no guarantees of success, following the Section 8.2 steps will maximize the chance for the earliest possible completion of RFCs suitable for expediting. 


\subsection{Normal processing}

\begin{tabular}{|c|c|}
\hline 1. Identify problem. & $\begin{array}{l}\text { Discuss problem with others who are qualified to propose solutions. Based on } \\
\text { discussion, determine if a Proposed Recommended Practice is appropriate in } \\
\text { addition to or instead of an RFC. Refer to the IGES Recommended Practices } \\
\text { Guide for procedures to propose a Recommended Practice. }\end{array}$ \\
\hline $\begin{array}{l}\text { 2. Initial Prepara- } \\
\text { tion }\end{array}$ & $\begin{array}{l}\text { Assuming an RFC should be proposed, obtain a number from the IGES } \\
\text { Change Control Secretary by submitting a paper RFC form containing a short } \\
\text { statement of the problem and proposed solution(s). Copy the RFC skeleton } \\
\text { file and complete author information, title, problem statement, and proposed } \\
\text { solution. Submit electronically as defined in } 3.2 .4 \text {. Alternatively, prepare a } \\
\text { similar-appearance form (see Appendix B of this document) with a word pro- } \\
\text { cessor, print, and submit the printed output. (Note: if not using the designated } \\
\text { Specification document processor, this work will have to be redone before the } \\
\text { RFC is eligible for mail ballot, so it may be better to use the copying method } \\
\text { initially.) When accepted by the Secretary, RFC is now "official"; it receives } \\
\text { Unassigned status. Use the RFC number for entity type or form numbers (see } \\
\text { section 3.2.1). }\end{array}$ \\
\hline 3. Routing RFC & $\begin{array}{l}\text { RFC will be forwarded to the IGES Project Committee for assignment to a } \\
\text { custodian Technical Committee and determination of the need for Gray Page } \\
\text { Testing. RFC authors may attend the IGES Project Committee meeting if } \\
\text { desired. When assigned to a custodian, RFC has Open status. }\end{array}$ \\
\hline $\begin{array}{l}\text { 4. Creating Fig- } \\
\text { ure(s) }\end{array}$ & $\begin{array}{l}\text { If any illustrations are required, prepare them as IGES files now. Choose full } \\
\text { page (portrait) or half page (landscape). File must be l-to-l scale. Minimum } \\
\text { text height is } 5 \mathrm{~mm} \text { ( } 0.2 \text { inches). Explanatory annotation should be } 6 \mathrm{~mm}(0.25 \\
\text { inches). Include only illustration, with no captions or outside border lines. Use } \\
\text { only } 2 \mathrm{D} \text { entities in the X-Y plane if possible. Use one file for each illustration. } \\
\text { Use ASCII (non-compressed). Save on floppy disk and label with name, RFC } \\
\text { number, operating system, and density. Obtain advance approval from IGES } \\
\text { Editor if non-standard illustrations (e.g., photos) are desired. }\end{array}$ \\
\hline 5. Sponsoring RFC & $\begin{array}{l}\text { RFC author should attend meeting of the custodian Technical Committee to } \\
\text { discuss the RFC and identify level of committee support. If the author does } \\
\text { not attend and the committee feels it cannot continue, RFC receives Tabled } \\
\text { status until continuation is possible; otherwise, the RFC is discussed and pos- } \\
\text { sibly amended. If committee rejects RFC, and author agrees to withdraw it, } \\
\text { RFC receives Withdrawn status and process ends. If committee rejects RFC } \\
\text { and author refuses to withdraw it, RFC receives Canceled status; author may } \\
\text { appeal to IGES Project Committee. If committee accepts RFC, it will retain } \\
\text { Open status while the author completes the next step. }\end{array}$ \\
\hline 6. Integrating RFC & $\begin{array}{l}\text { Prepare "integrated electronic form" of the RFC to make it "ballot-ready." } \\
\text { Obtain electronic masters of the RFC form and the appropriate section of the } \\
\text { Specification from the IGES Editorial Committee; these will be in the input } \\
\text { format of designated document processor for the Specification (currently } \\
\text { LAT }_{E} \mathrm{X} \text { ). Edit as required. If using illustrations, process using designated IGES } \\
\text { post-processor for the Specification (see section 3.2.4). Print copies of RFC } \\
\text { and IGES files for review by the IGES Editorial Committee. After review, } \\
\text { make corrections as required to electronic input and reprint. Save on floppy } \\
\text { disk (see rules in step 4). }\end{array}$ \\
\hline
\end{tabular}




\begin{tabular}{|c|c|}
\hline 7. Promoting RFC & $\begin{array}{l}\text { Author returns to custodian Technical Committee meeting with the "inte- } \\
\text { grated electronic form" RFC and paper copies which have been reviewed and } \\
\text { approved by the IGES Editorial Committee. If custodian committee decides } \\
\text { RFC must be significantly changed, steps } 5,6 \text {, and } 7 \text { are repeated until satis- } \\
\text { factory. When custodian committee accepts RFC (or makes minor changes), } \\
\text { RFC receives Recommended for Ballot status; custodian committee chair- } \\
\text { person delivers RFC to the IGES Project Committee for oversight approval. }\end{array}$ \\
\hline 8. Project Review & $\begin{array}{l}\text { IGES Project Committee meets to review RFCs having Recommended for } \\
\text { Ballot status; author may attend the meeting. If RFC meets "ballot-ready" } \\
\text { requirements, RFC receives Approved for Ballot status. IGES Project Com- } \\
\text { mittee delivers RFC to the IGES Ballot Coordinator; otherwise, RFC receives } \\
\text { Reviewed status and is returned to step } 6 .\end{array}$ \\
\hline 9. Ballot RFC & $\begin{array}{l}\text { RFC is considered by members of the IGES Ballot Group. Ballot results and } \\
\text { comments from the voting process are returned to the IGES Project Commit- } \\
\text { tee; RFC receives Balloted status. Any RFC receiving only "Approve" votes } \\
\text { and no comments receives ECO status. If Gray Page Testing is required but } \\
\text { not completed, the entity receives untested designation; otherwise, it becomes } \\
\text { part of the Specification. In most cases, disapprovals and comments will } \\
\text { occur; these are forwarded to the RFC author. In addition, they are separated } \\
\text { into distinct thoughts, entered into a comments database, and printed on Com- } \\
\text { ment Disposition Forms for delivery to the Chairperson of the custodian Tech- } \\
\text { nical Committee. All ballot comments must be considered and resolved. }\end{array}$ \\
\hline $\begin{array}{l}\text { 10. Comment han- } \\
\text { dling }\end{array}$ & $\begin{array}{l}\text { Between IPO meetings, RFC author personally contacts each commentor in } \\
\text { an attempt to resolve their comments before the next meeting; ordinarily, this } \\
\text { succeeds for minor objections and the author amends the integrated form RFC } \\
\text { and prints new copies. If the author does not succeed in resolving all com- } \\
\text { ments, the comments will be handled at the next IPO meeting. The author is } \\
\text { responsible for reporting all results of attempted comment resolution to the } \\
\text { IGES Change Control Secretary. }\end{array}$ \\
\hline 11. TC review & $\begin{array}{l}\text { Custodian Technical Committee meets to review advance comment resolution } \\
\text { by RFC author and to handle unresolved comments. The Comment Disposi- } \\
\text { tion Form (as printed from the comment database) must be used to document } \\
\text { comment resolution. If comment resolution causes significant editorial } \\
\text { changes, review them with the IGES Editorial Committee. If comment resolu- } \\
\text { tion identifies that the RFC cannot be amended, committee decides if salvage } \\
\text { should be attempted; if so, RFC receives Open status and process returns to } \\
\text { step 3. The author will be asked to withdraw an unsalvageable RFC; if author } \\
\text { agrees, RFC receives Withdrawn status and process ends. If author refuses to } \\
\text { withdraw it, RFC receives Cancelled status; author may appeal to IGES } \\
\text { Project Committee. If comment resolution causes change in technical intent, } \\
\text { RFC receives Recommended for Ballot status and process returns to step 8; } \\
\text { otherwise, RFC receives Recommended for Gray Pages status (if testing } \\
\text { required, but not completed) or Recommended for Update status (if testing } \\
\text { is successfully completed). Results are delivered to the IGES Project Commit- } \\
\text { tee. }\end{array}$ \\
\hline
\end{tabular}




\begin{tabular}{|l|l|}
\hline 12. Project review & $\begin{array}{l}\text { IGES Project Committee meets to review RFCs having Recommended for } \\
\text { Gray Pages or Recommended for Update status. Minor editorial changes } \\
\text { may occur during review. If review is unsatisfactory, RFC receives Reviewed } \\
\text { status and process returns to step 11. If review is satisfactory, RFC receives } \\
\text { ECO status with a directive to update the Specification as appropriate. RFC is } \\
\text { delivered to the IGES Change Control Secretary for archiving. Secretary for- } \\
\text { wards RFC Cover Sheet and "integrated electronic form" of RFC to IGES } \\
\text { Editor upon completion of integrating minor editorial changes made in steps } \\
11 \text { or 12 which have not been integrated due to time constraints. }\end{array}$ \\
\hline $\begin{array}{l}\text { 13. Incorporation } \\
\text { by Editor }\end{array}$ & $\begin{array}{l}\text { IGES Editor uses ECO to update master copy of Specification using "inte- } \\
\text { grated electronic form" of RFC as part of document management procedure. }\end{array}$ \\
\hline
\end{tabular}




\subsection{Expedited processing}

\begin{tabular}{|c|c|}
\hline 1. Identify problem & $\begin{array}{l}\text { Discuss problem with others who are qualified to propose solutions. Based on } \\
\text { discussion, determine if a Proposed Recommended Practice is appropriate in } \\
\text { addition to or instead of an RFC. Refer to the IGES Recommended Practices } \\
\text { Guide for procedures to propose a Recommended Practice. Before continuing, } \\
\text { you should be certain there is support in the Technical Committee which is the } \\
\text { obvious custodian, otherwise, expediting will be fruitless. }\end{array}$ \\
\hline 2. Integrating RFC & 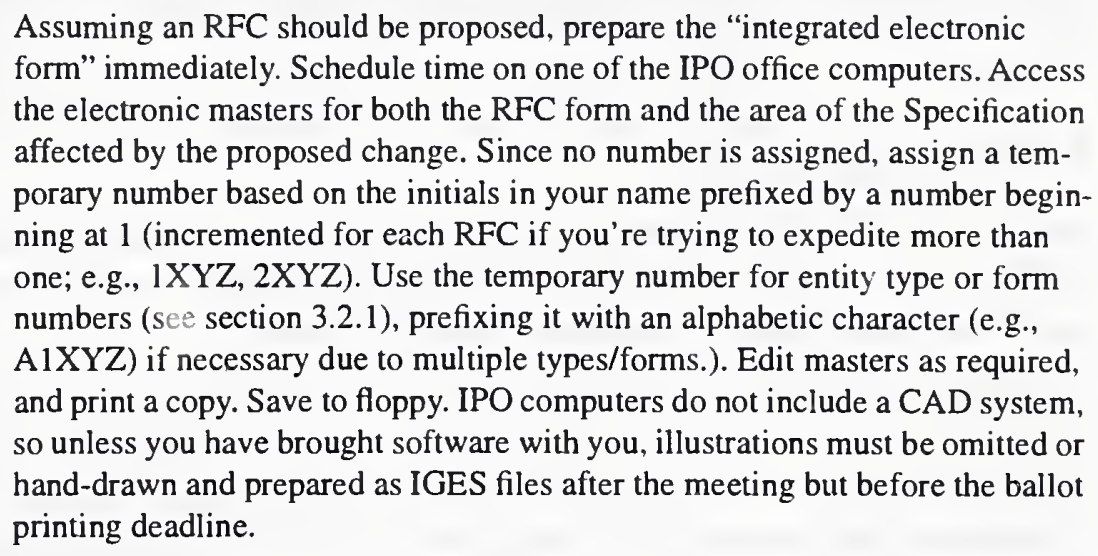 \\
\hline 3. Project Review & $\begin{array}{l}\text { Deliver printed copy to IGES Editorial Committee. Request expedited review. } \\
\text { If possible, a meeting time for review will be scheduled on-the-spot. Use IPO } \\
\text { computer again to make corrections as required. }\end{array}$ \\
\hline 4. TC Process & $\begin{array}{l}\text { RFC author must attend meeting of the obvious custodian Technical Commit- } \\
\text { tee to discuss the RFC. If committee support was identified as recommended } \\
\text { in step 1, the RFC should be ready to go "as is" or with minor amendment. If } \\
\text { committee votes against the RFC, expediting has failed; RFC author must } \\
\text { withdraw it or resume normal processing at step } 2 \text { (see Section } 8.1 \text { ). If the } \\
\text { committee votes for the RFC, it receives Recommended for Ballot status. }\end{array}$ \\
\hline 5. Project Review & $\begin{array}{l}\text { Technical Committee Chairperson attends meeting of the IGES Project Com- } \\
\text { mittee. The author may attend, and is encouraged to do so. Chairperson } \\
\text { requests expedited RFC processing. The IGES Change Control Secretary } \\
\text { assigns a permanent RFC tracking number immediately. If request for expe- } \\
\text { diting is approved, the IGES Project Committee performs oversight review } \\
\text { immediately. If RFC meets "ballot-ready" requirements, the RFC receives } \\
\text { Approved for Ballot status; this status may be conditional upon completion } \\
\text { of minor editorial corrections or upon delivery of IGES files for illustrations to } \\
\text { the IGES Ballot Coordinator. If major problems are found, or if request for } \\
\text { expediting is denied, expediting has failed; RFC receives Open status and } \\
\text { returns to normal processing at step } 7 \text { (see Section 8.1). }\end{array}$ \\
\hline 6. Final steps & $\begin{array}{l}\text { Expediting has succeeded; RFC returns to normal processing at Step } 9 \text { (see } \\
\text { Section } 8.1 \text { ). After ballot results are received, author must make a special } \\
\text { effort to resolve all comments before the next IPO meeting (see step } 10 \text {, Sec- } \\
\text { tion } 8.1 \text { ) to prevent comment resolution delays from preventing the RFC from } \\
\text { getting into the desired version of the Specification. }\end{array}$ \\
\hline
\end{tabular}




\subsection{Editing, Managing, and Publishing the Specification}

The IGES Editor and IGES Editorial Committee, along with Technical Committees and RFC authors proposing changes, jointly share the responsibility for producing the Specification in a high-quality manner. It should be written succinctly, grammatically correct, organized in an easyto-use manner, consistent in style, and accurately managed to avoid introducing errors.

The IGES Editor is responsible for ensuring that Editorial Committee members are qualified to perform the editorial review required to attain these goals.

\subsection{Editorial Review}

Neither the IGES Editor nor the Editorial Committee members are responsible for preparing RFCs. If either chooses to make an exception and help someone out, this shall not be interpreted as setting a precedent for future agreement to do so.

All RFCs must undergo "editorial review" during their preparation process. Any member of the IGES Editorial Committee may perform the review. If the RFC author disagrees with any Editorial Committee member's review, the author may request a secondary review by the IGES Editor. If the secondary review still is unsatisfactory, the RFC author may appeal to the IGES Project Committee (see Section 5).

In some instances, the amendment of the RFC during consideration by the custodian Technical Committee, the IGES Project Committee, and by the IGES Ballot Group may require repeated editorial reviews until the RFC reaches ECO status.

Editorial review results in marking up the paper copy of the RFC by the reviewer. The RFC author (or if unavailable, the Chairperson of the custodian Technical Committee) is responsible for making the exact corrections marked to the electronic form of the RFC. If inexact corrections are made, the IGES Editor reserves the right to correct them during the document update process or to return the ECO to the IGES Project Committee for reconsideration.

The IPO shall provide funding as necessary for reasonable expenses incurred by the IGES Editor for editing and producing of the Specification.

\subsection{Managing the document}

The IGES Editor is a volunteer position; therefore, it is likely that his employer's computer resources will be used for managing the configuration of the Specification document. Accordingly, the Editor may choose any workable methods to accomplish this task which are available and suitable.

Ordinary care shall be exercised in the security of the data, and at least two off-site back-up copies of relevant data shall be retained in different locations. Data to be retained includes: the master files for the Specification, the designated document processor software, and the designated IGES post-processor software. Regular on-site backups should be performed.

The input used to update the Specification (i.e., the integrated electronic form RFC) shall be retained permanently on magnetic media or until such time as further changes to the Specification are no longer made. 


\subsection{Publishing the document}

The IGES Editor shall prepare camera-ready output of the Specification when directed to do so by the IGES Project Committee in response to its assignment of Approval for Ballot of a tracking RFC to approve a new version of the Specification, and at other times as necessary, such as submission of the Specification to ANSI as a proposed national standard.

Publishing of the Specification is the joint responsibility of US Pro and NIST. Any agency distributing copies of the Initial Graphics Exchange Specification is hereby directed by the IGES Project Committee to include the applicable IGES Recommended Practices Guide with all copies of the Specification either sold or given away since any Specification user should have access to both documents; the Guide may be distributed separately, but not the Specification. 



\section{Appendix A A ctivity Model: Changing IGES}

The following model is : ne IDEF0 language. The reference for this language is FIPS 183. This FIPS may be accessed through Internet World Wide Web (http://nemo.ncsl.nist.gov/idef); a copy of this standard may be obtained by request for FIPSPUB 183 to

\section{NTIS}

U.S. Department of Commerce

Springfield, VA 22161

\section{Model Sheets:}

A-0; The Procedures for Changing IGES

A 0; Make Repair or Extension to the Specification

A 0 Text; Description of Activities

A 2; Draft an RFC

A 2 Text; Description of Activities

A 24; Create $\mathrm{IAT}_{\mathrm{E}} \mathrm{X}$ RFC

A 24 Text; Description of Activities

A 4; Ballot the RFC

A 4 Text; Description of Activities 



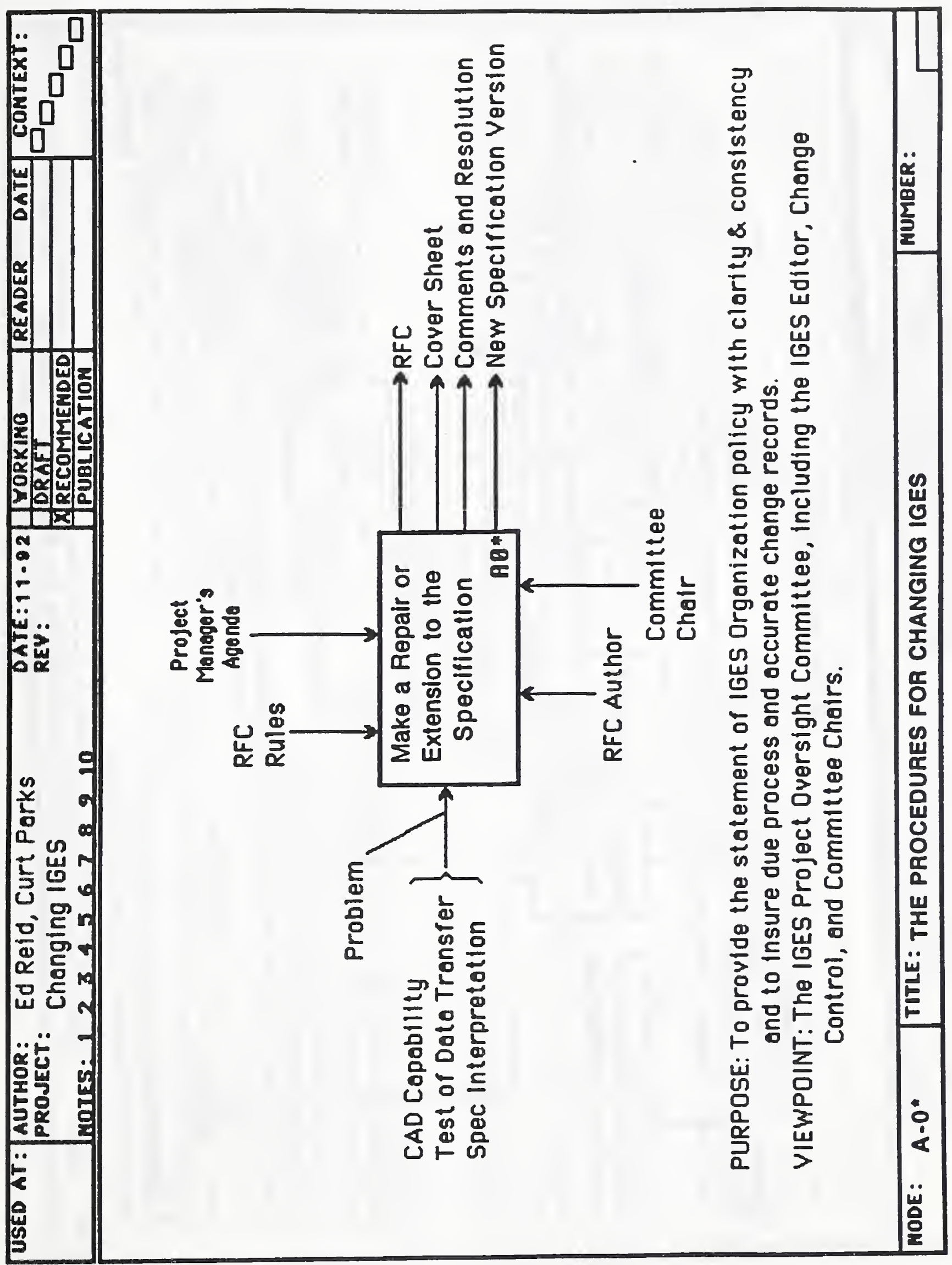




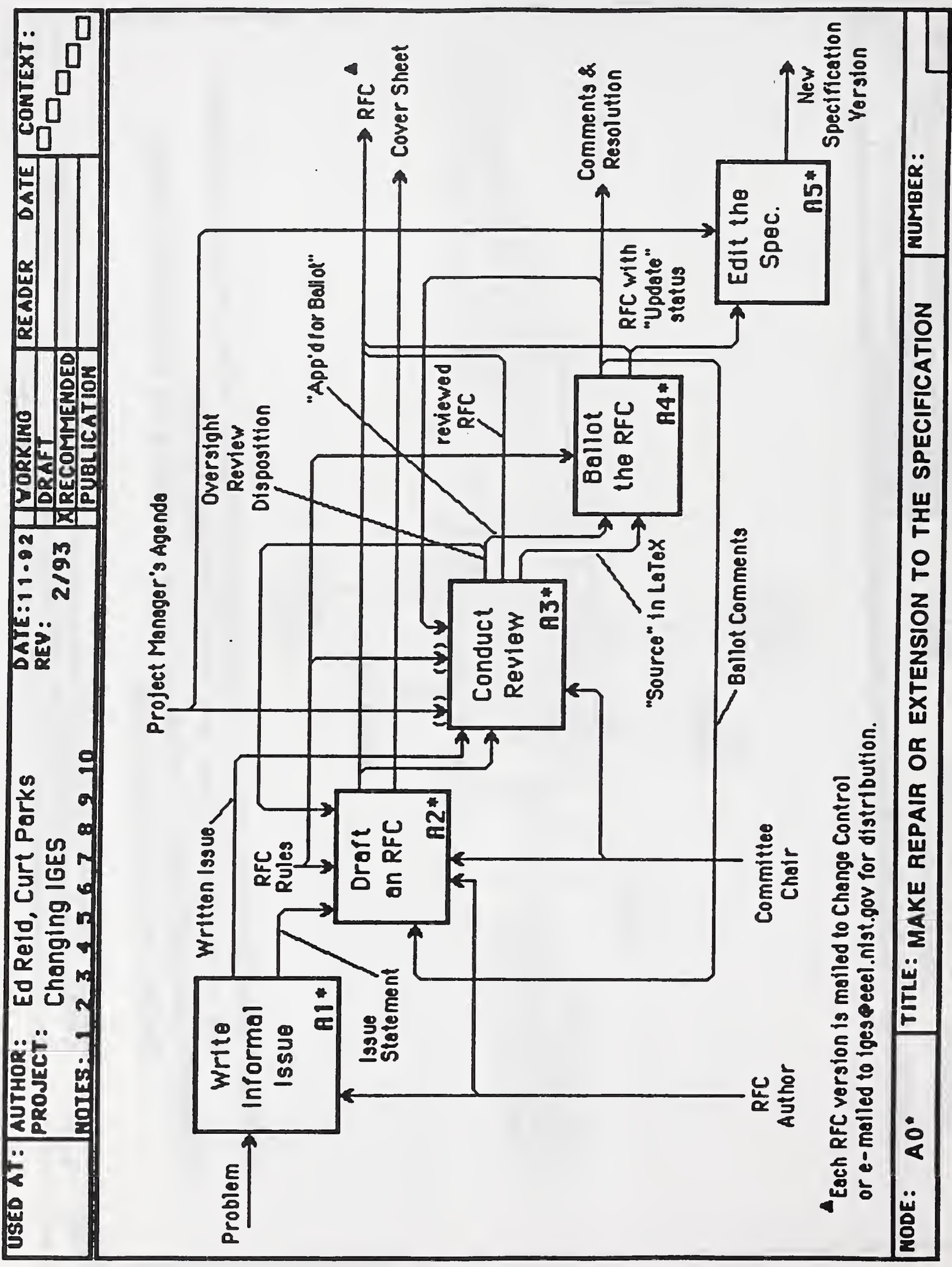




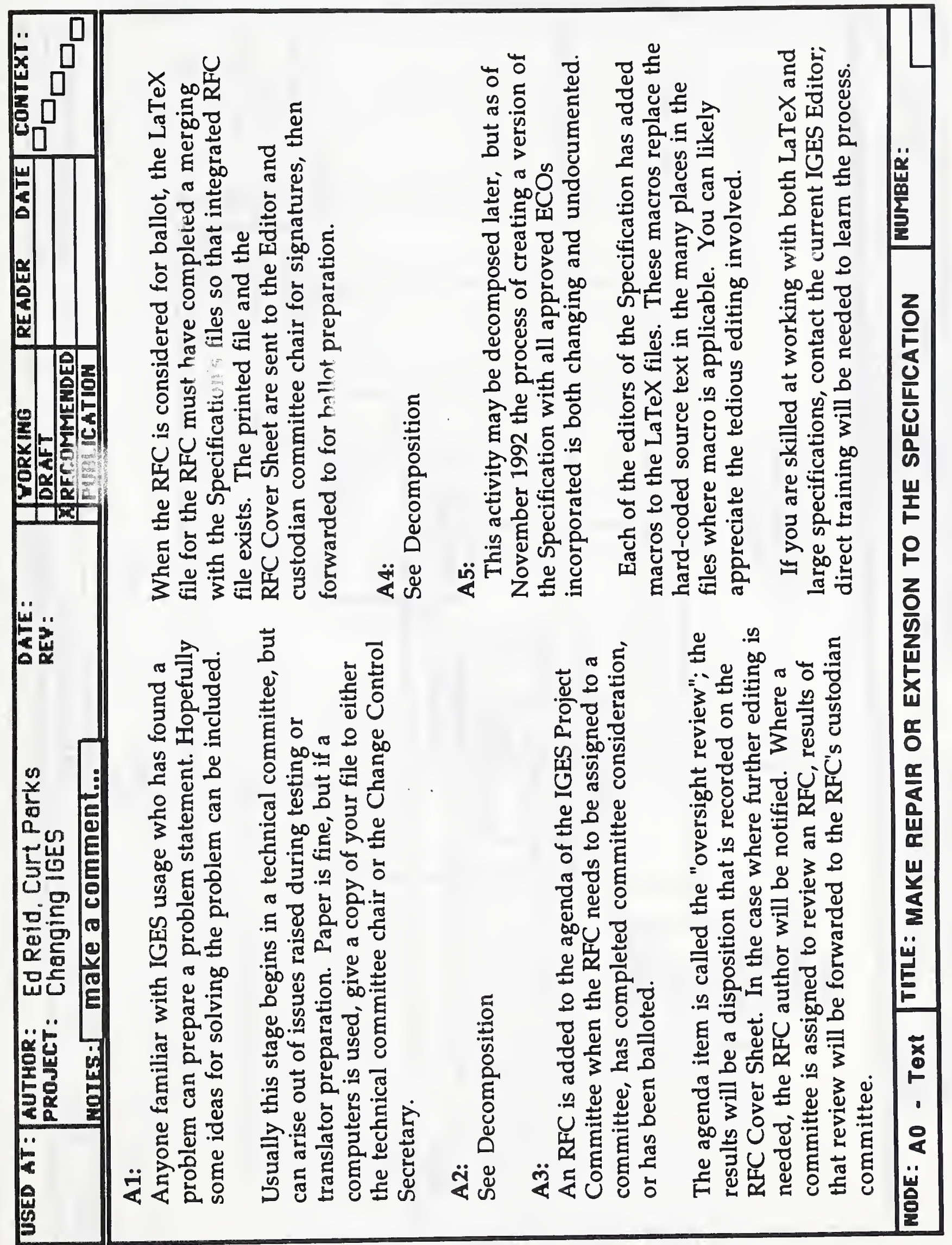




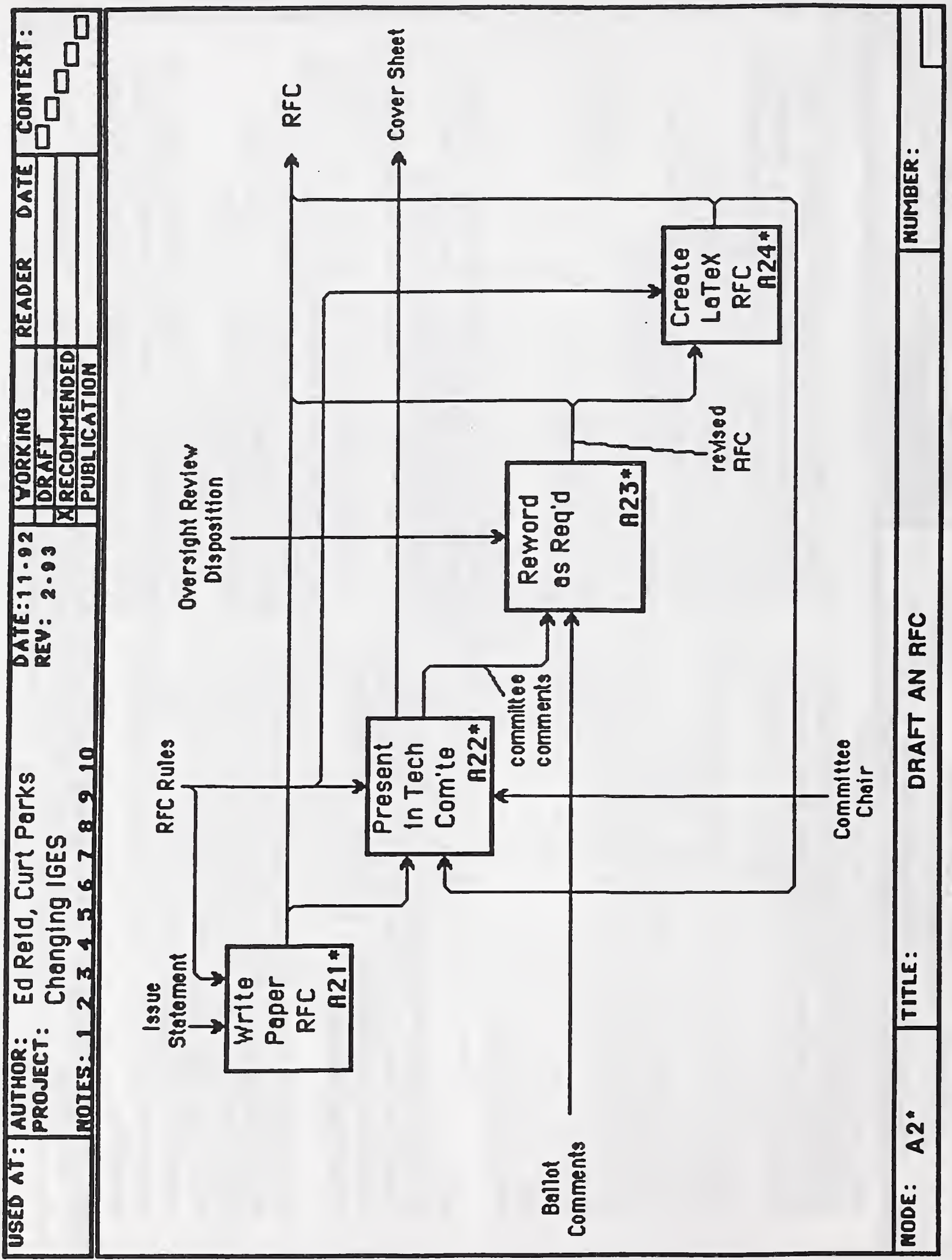




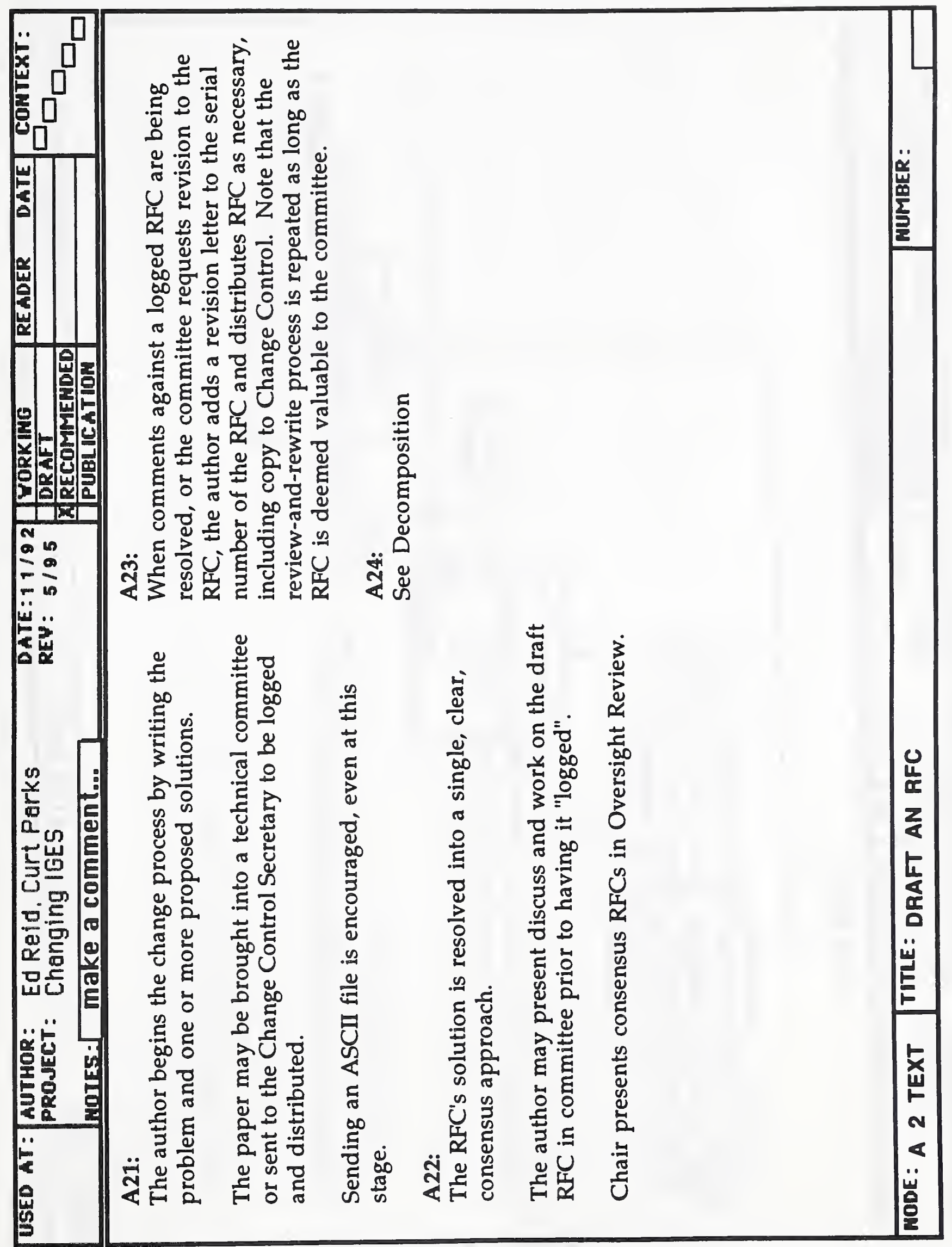




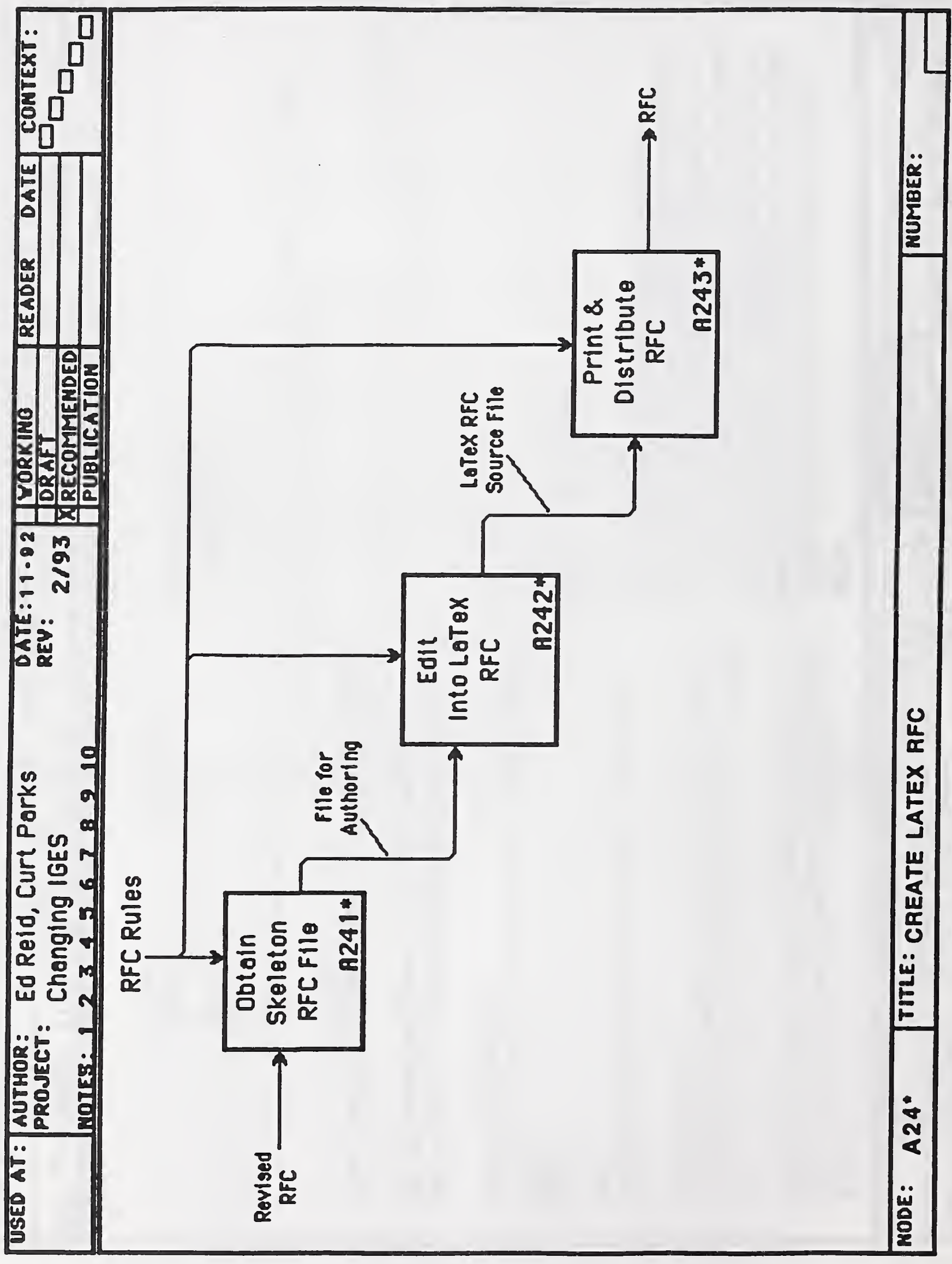




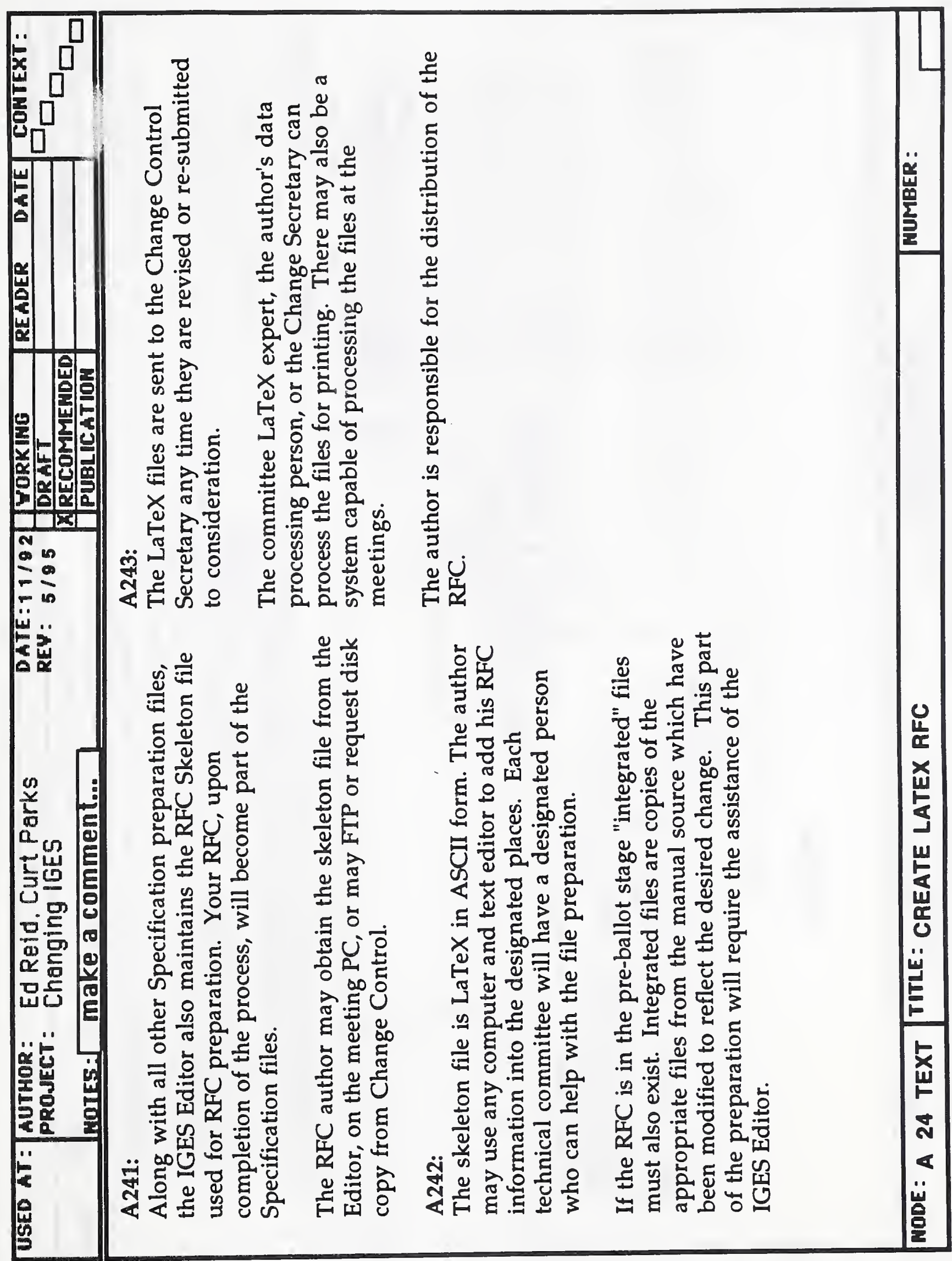




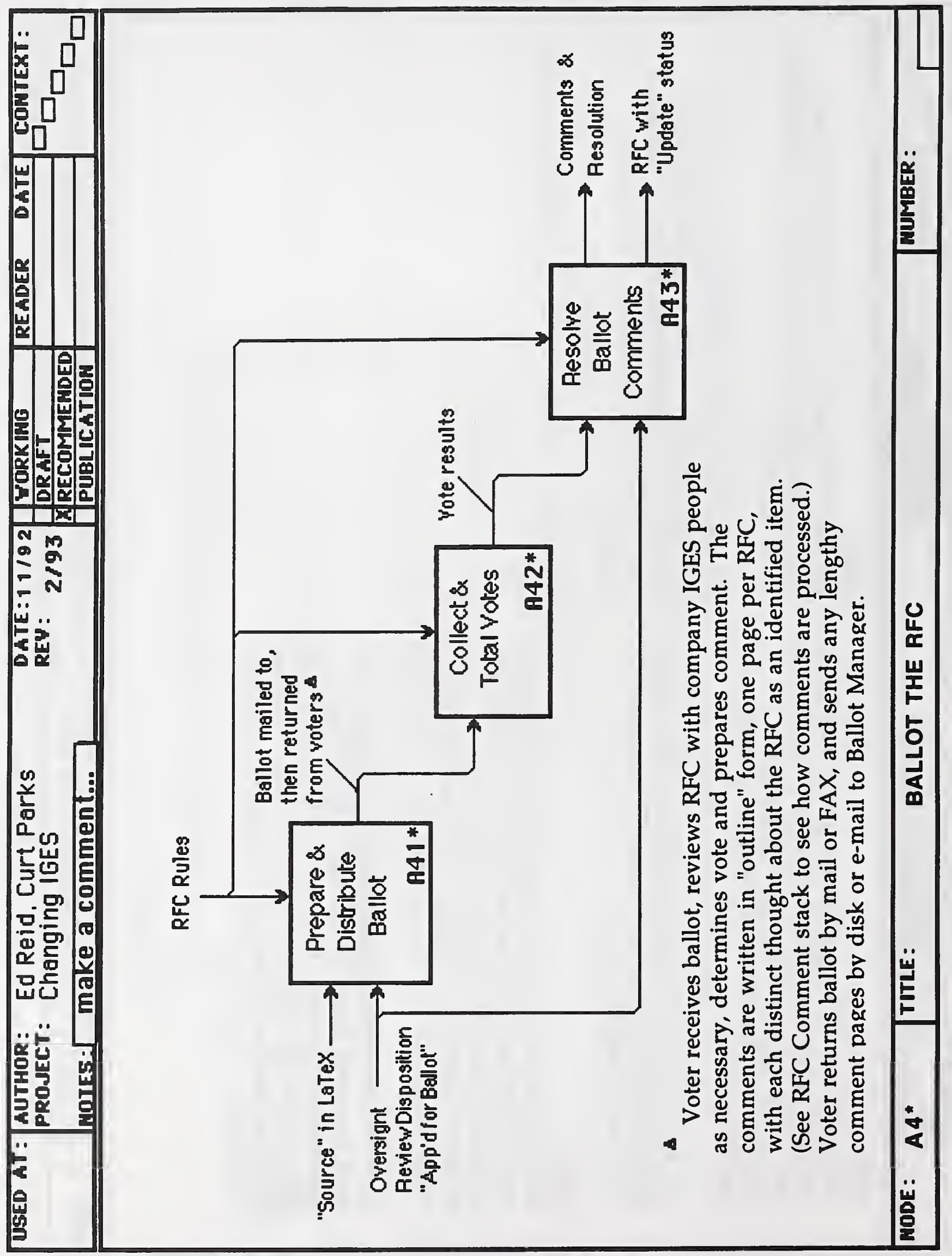




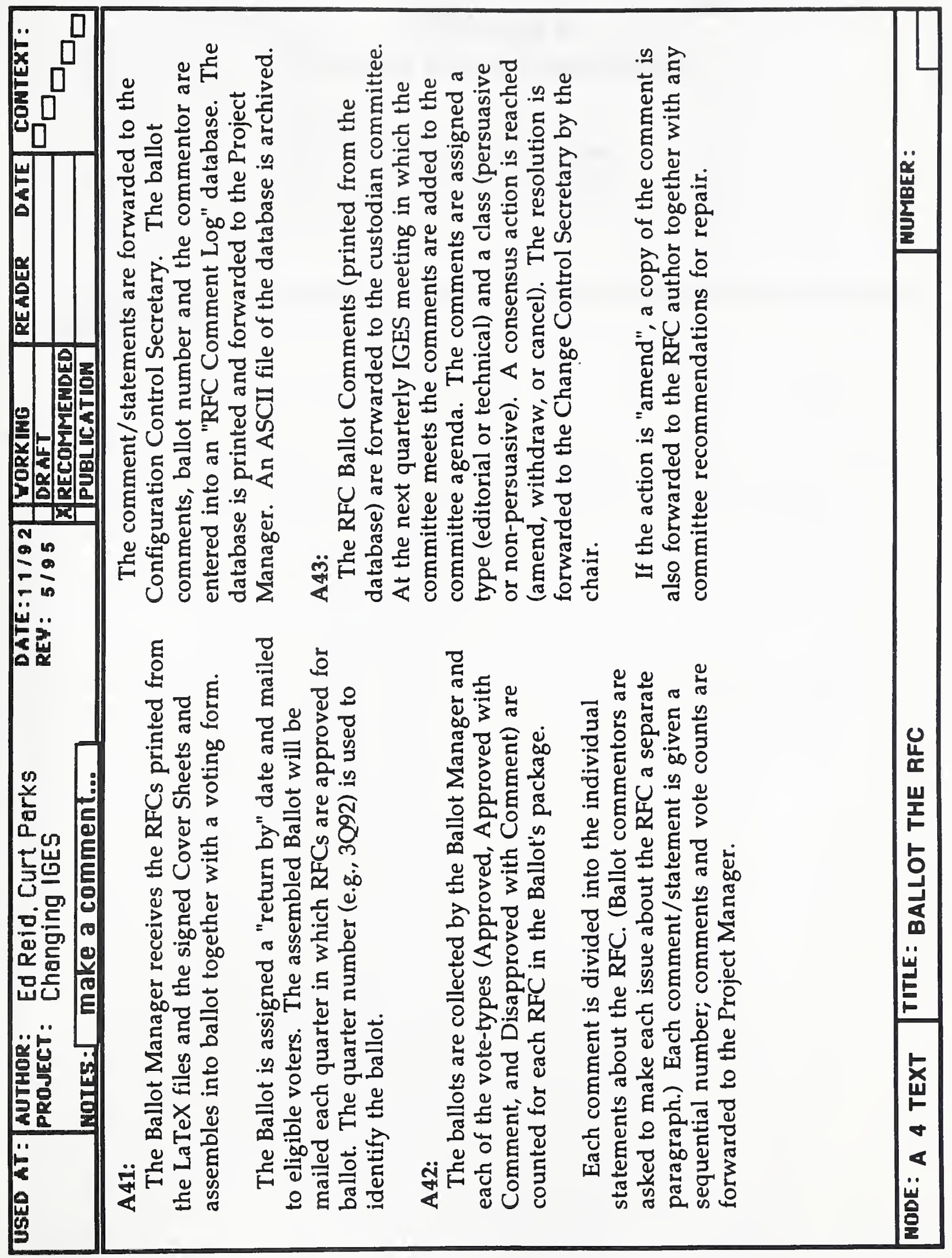





\title{
Appendix B \\ Example Cover Sheet Forms
}

\author{
Contents \\ Request for Change (RFC) example \\ Edit Change Order (ECO) example*
}

* NOTE: Example only; the form depicted herein is restricted to being printed from the integrated IGES Source files. 


\section{IGES Request for Change}

RFC Number:

Revision:

Rev. Date:

Title:

Date of Receipt:

Custodian TC:

Gray page testing required:

Author:

Phone:

FAX:

E-Mail:

Approval for Ballot:

Date:

Problem Description

Proposed Solution 


\section{IGES Edit Change Order}

ECO Number:

Reference RFC Number:

Title:

Grey page testing required?

Approvals for Release

Committee Chairman

Date:

Date:

IGES Project Manager

Effect of ECO: 

